Revista Brasileira de Meteorologia, v.23, n.4, 381 - 403, 2008

\title{
ESTUDO DA ENERGÉTICA MODAL PARA EPISÓDIOS DE ZCAS. PARTE II: IMPACTO DA RESOLUÇÃO DO MODELO E DA PARAMETRIZAÇÃO DE CONVECÇÃO
}

\author{
RENATA WEISSMANN BORGES MENDONÇA e JOSÉ PAULO BONATTI
}

\author{
Instituto Nacional de Pesquisas Espaciais (INPE) \\ Centro de Previsão de Tempo e Estudos Climáticos (CPTEC) \\ renata.mendonca@cptec.inpe.br, \\ paulo.bonatti@cptec.inpe.br
}

Recebido Novembro 2007 - Aceito Setembro 2008

\begin{abstract}
RESUMO
O desempenho do Modelo Global do Centro de Previsão de Tempo e Estudos Climáticos (CPTEC) em simular a energética modal para um composto de sete episódios de Zona de Convergência do Atlântico Sul (ZCAS) é avaliado, enfatizando-se a influência da resolução espacial do modelo e de três diferentes parametrizações de convecção profunda: Kuo, Relaxed Arakawa-Schubert (RAS) e Grell na partição vertical de energia entre os modos externos e internos, e as trocas de energia entre os modos horizontais de oscilação Rossby, Kelvin, Misto Rossby-Gravidade, Gravidade Oeste e Leste. Os resultados mostraram que as previsões utilizando os esquemas de convecção profunda Kuo, RAS e Grell foram semelhantes entre si e apresentaram uma boa concordância em relação aos padrões obtidos na parte observacional (Parte I deste artigo). O emprego de diferentes esquemas de convecção profunda não apresentou impactos significativos na partição e interação de energia entre os modos verticais e horizontais. Um impacto maior foi obtido com o aumento da resolução vertical das análises e do modelo, de 28 para 42 níveis, em que um maior número de modos internos apresenta um papel relevante nas trocas horizontais e verticais de energia.
\end{abstract}

Palavras Chave: Energética, Modos Normais, Zona de Convergência do Atlântico Sul (ZCAS), Modelo de Circulação Geral Atmosférico.

ABSTRACT: STUDY OF THE MODALENERGETICS FOR SACZ EPISODES. PART II: IMPACT
OF THE MODEL RESOLUTION AND THE CONVECTION PARAMETERIZATION
The performance of the CPTEC Global Model in simulating the modal energetics for a composite of
seven South Atlantic Convergence Zone (SACZ) episodes was evaluated, emphasizing the influence
of the model resolution and the three different deep convection parameterizations: Kuo, Relaxed
Arakawa-Schubert (RAS) and Grell on the vertical energy partition between external and internal
modes and on the energy interactions within and between various horizontal oscillation modes:
Rossby, Kelvin, Mixed Rossby-Gravity and West and East Gravity. The results showed that the model
predictions using the Kuo, RAS and Grell deep convection schemes were similar with each other,
and had a good agreement with the patterns obtained in the observational part (Part I of this paper).
The use of different deep convection schemes did not present significant impact in the partition and
interaction of energy between vertical and horizontal modes. A greater impact was obtained when
increasing the vertical resolution of the analyses and the model from 28 to 42 levels. A greater number
of internal modes show a relevant role in the horizontal and vertical energy exchanges, in terms of
representing the observed characteristics. Keywords: Energetics, normal mode functions, South Atlantic Convergence Zone (SACZ), Atmospheric General Circulation Model. 


\section{INTRODUÇÃO}

A existência de uma interação entre a convecção tropical e os sistemas de grande escala é conhecida desde a década de 60, a partir de diversos estudos observacionais (Malkus e Williams, 1963; Matsumoto et al., 1967; Cho e Ogura, 1974). As interações entre o aquecimento devido à condensação de vapor d'água e a dinâmica da atmosfera conduzem a efeitos em diversas escalas espaciais e temporais. O processo de condensação é iniciado e modulado pela dinâmica de grande escala, enquanto o aquecimento latente fornece uma importante forçante para a dinâmica de grande escala. Esta forçante é, portanto, crucial para determinar a estrutura da circulação atmosférica nas latitudes tropicais e médias (Straus e Huntley, 1994).

Representar o processo de liberação de calor latente associada à convecção profunda em modelos numéricos de previsão de tempo é uma tarefa difícil, uma vez que há um conhecimento limitado de como este processo realmente ocorre na natureza e ao fato de que a interação entre a convecção e a circulação de grande escala não é linear.

Nas últimas décadas, a interação da circulação de grande escala e a convecção cumulus tem recebido especial atenção, principalmente no que diz respeito aos esquemas que a parametrizam (Kuo et al., 1997). Diversos esquemas, Kuo (1974), Arakawa e Schubert (1974), Kreitzberg e Perkey (1976), Fritsch e Chappell (1980), Betts (1986) e Betts-Miller (1986), Kain e Fritsch (1990) e Grell (1993), etc., foram desenvolvidos e incorporados em modelos numéricos, contudo nenhum dos esquemas existentes pode ser considerado como teoria geral para o amplo conjunto de situações atmosféricas (Kuo et al., 1997; Wang e Seaman, 1997).

Uma das características da circulação regional, dominante durante o verão da América do Sul, é a Zona de Convergência do Atlântico Sul (ZCAS). A ZCAS, freqüentemente observada em imagens de satélite, é convencionalmente definida como uma faixa persistente, pelo menos 4 dias seguidos (Quadro, 1994), de nebulosidade e precipitação, com orientação noroeste-sudeste, que se estende desde o sul da região Amazônica até o Atlântico Sul-Central, cuja estrutura é originada e mantida pela interação entre a convecção tropical e os sistemas de grande escala.

Muitos trabalhos (Kodama, 1992 e 1993; Grimm e Silva Dias, 1995; Figueroa, 1997; Gandu e Silva Dias, 1998; Carvalho et al., 2004, etc) enfatizam o importante papel de forçantes térmicas associadas à fontes de calor representadas pela liberação de calor latente na Amazônia e Indonésia, e suas extensões para sudeste associadas à ZCAS e ZCPS, respectivamente. Entretanto, muitos dos aspectos discutidos não são considerados em estudos de previsibilidade, os quais, se incluídos, poderiam contribuir para aumentar significativamente a escala de tempo de validade das previsões, especialmente a previsão de tempo e clima em regiões tropicais e subtropicais como a do Brasil, que são influenciadas por sistemas, nos quais a liberação de calor latente tem papel significativo em suas energéticas.

Dentro deste contexto, o objetivo principal deste trabalho é avaliar o desempenho do modelo de circulação geral atmosférico do Centro de Previsão de Tempo e Estudos Climáticos (MCGA-CPTEC) para o cálculo da energética modal para episódios de ZCAS, a fim de verificar o impacto da utilização de diferentes resoluções espaciais e parametrizações de convecção profunda na partição e interação de energia entre os modos verticais (externos e internos) e horizontais de oscilação, ou seja, analisar a capacidade do modelo global do CPTEC em reproduzir a partição de energia observada.

$\mathrm{Na}$ seção 2 são descritos os dados e a metodologia utilizada, bem como os episódios selecionados para este estudo. Os resultados da análise energética são apresentados e discutidos na seção 3. As conclusões são apresentadas na seção 4.

\section{DADOS E METODOLOGIA}

\subsection{Dados}

Os dados utilizados são as análises do National Centers for Environmental Predictions (NCEP), com resoluções T126L28 e T170L42, onde Tttt significa truncamento triangular na onda zonal ttt e Lll significa 11 camadas verticais em coordenadas sigma, e horário das $12 \mathrm{Z}$ (daqui em diante, referenciadas como análise T126L28 e análise T170L42, respectivamente). Essas análises contêm campos espectrais de topografia, logaritmo natural da pressão à superfície, temperatura virtual, divergência horizontal do vento, vorticidade e umidade específica. As previsões numéricas, obtidas das rodadas do MCGA-CPTEC a partir das condições iniciais do NCEP, são consideradas para analisar a capacidade do modelo global em reproduzir a partição de energia em modos verticais e horizontais observada, apresentada na Parte I deste artigo (Mendonça e Bonatti, 2008, daqui para frente referenciado como MB08). Estimativas de precipitação diária provenientes do Global Precipitation Climatology Project (GPCP) são utilizadas para ilustrar e comparar os campos de precipitação e de partição de energia, associados ao composto dos episódios de ZCAS selecionados.

\subsection{Modelo de Circulação Geral Atmosférico do CPTEC}

O MCGA-CPTEC foi originalmente desenvolvido no NCEP e posteriormente modificado pelo Center for OceanLand-Atmosphere Studies (COLA/USA), nas décadas de 


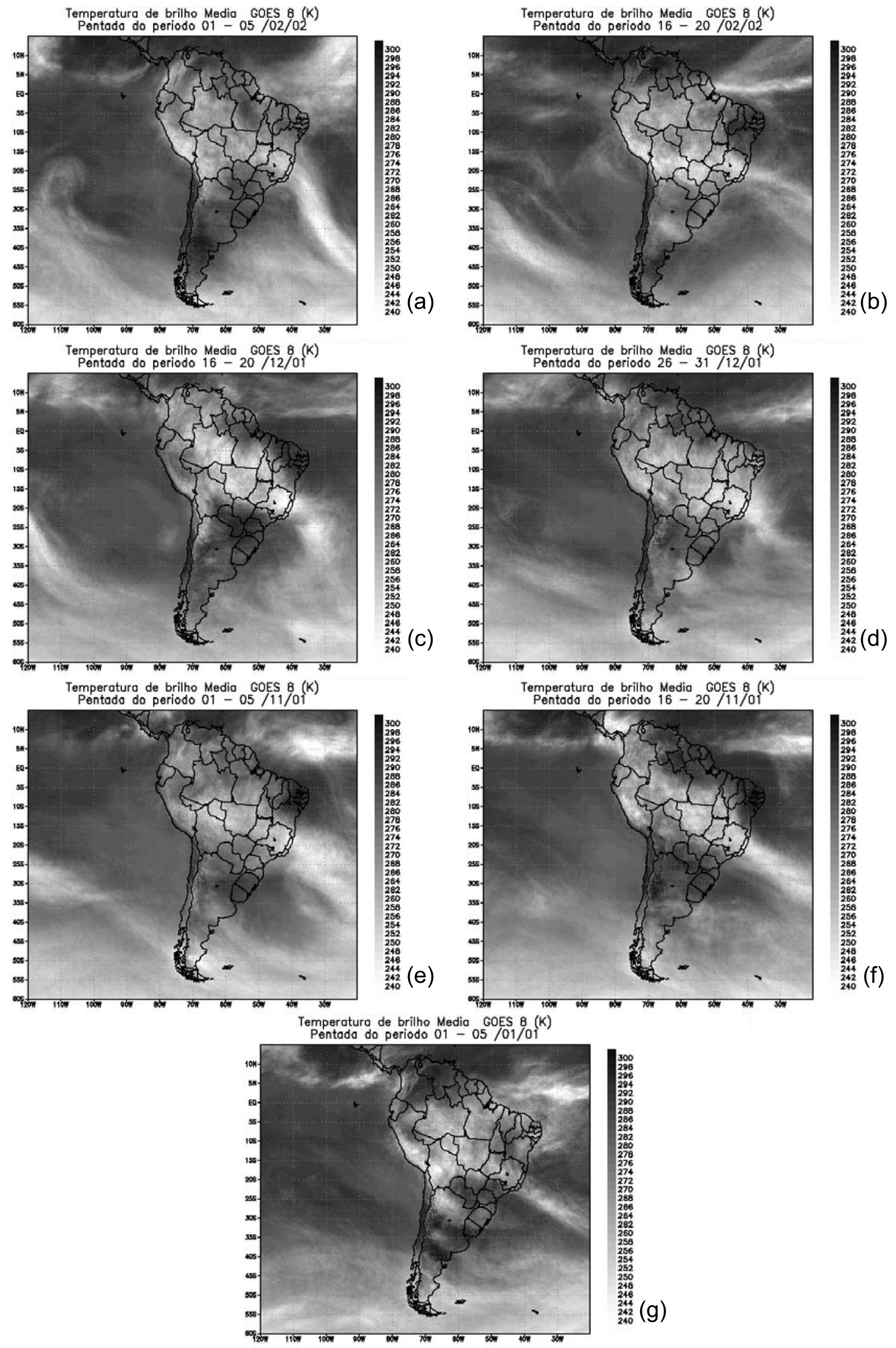

Figura 1 - Temperatura de Brilho Média para pêntadas referentes aos episódios de ZCAS selecionados. FONTE: Metop/CPTEC/INPE. 
80/90, e pelo CPTEC nos últimos 10 anos, sendo utilizado operacionalmente tanto para previsão de tempo, quanto para previsão climática sazonal. A parte dinâmica do modelo usa as equações espectrais primitivas em forma de divergência e vorticidade, temperatura virtual, umidade específica e logaritmo natural da pressão à superfície. Os processos físicos são considerados no modelo através de parametrizações físicas. Maiores detalhes sobre a descrição do modelo, bem como dos esquemas de parametrizações físicas utilizados podem ser encontrados em Bonatti (1996) e Kinter et al. (1997).

\subsection{Episódios Selecionados}

O estudo é realizado para um composto de 7 episódios de ZCAS ocorridos nos períodos de janeiro, novembro e dezembro de 2001, e fevereiro de 2002, conforme descrição do Boletim Climanálise (2001a, b e c, 2002). Estes episódios são os mesmos utilizados na análise diagnóstica da energética apresentada na Parte I deste artigo.

As imagens de satélite apresentadas na Figura 1, mostram a temperatura de brilho média para as pêntadas referentes aos períodos (ou parte dos períodos) de atuação destes episódios de ZCAS e indicam o posicionamento da banda de nebulosidade associada a cada um dos episódios selecionados.

\subsection{Análise da Partição Modal de Energia}

Para a análise da partição de energia em modos verticais e horizontais, é feita a recomposição das previsões espectrais no espaço físico, o cálculo das variáveis vento zonal, vento meridional e geopotencial generalizado $\left(\mathrm{P}=\phi-\bar{\phi}(\sigma)+\mathrm{R}_{\mathrm{d}} \overline{\mathrm{T}}(\sigma)\right.$ q, onde $\bar{\phi}(\sigma)$ é o geopotencial hidrostático em relação a $\mathrm{T}(\sigma)$, que é o perfil de temperatura da atmosfera básica, função apenas da coordenada vertical), e então é realizada a decomposição desses campos em modos verticais obtidos em coordenadas sigma e modos horizontais Rossby, Kelvin, Misto RossbyGravidade e Gravidade Oeste e Gravidade Leste, conforme descrito em Kasahara e Puri (1981) e ilustrado na Figura 1 da Parte I-Análise Observacional (artigo que acompanha este). Neste caso, os modos verticais são os mesmos que os do modelo global do CPTEC. A partir daí é calculada a energia em cada modo e sua porcentagem em relação ao total.

As equações (1) e (2) representam as contribuições para a energia total em cada modo vertical e horizontal, respectivamente:

$$
P_{n}=\frac{E_{C_{n}}+E_{P_{n}}}{\sum_{n=0}^{N}\left(E_{C_{n}}+E_{P_{n}}\right)} \times 100 \%
$$

onde $\mathrm{Ec}_{\mathrm{n}}, \mathrm{Ep}_{\mathrm{n}}$ representam, respectivamente a energia cinética e a energia potencial disponível associada a cada modo vertical $n$.

$$
\mathrm{PE}_{w}=\frac{\sum_{n=n_{1}}^{n_{2}} E_{w}}{\sum_{n=1}^{M} E_{T n}} \times 100 \%
$$

onde

$$
\mathrm{E}_{\mathrm{Tn}}=\mathrm{E}_{\text {Tsoma }}+\mathrm{E}_{\text {Tint }} \text {, }
$$

$$
\begin{aligned}
& \mathrm{E}_{\text {Tint }}=\mathrm{ET}_{\mathrm{RBKV}}+\mathrm{ET}_{\mathrm{RBMX}}+\mathrm{ET}_{\mathrm{RBGO}}+ \\
& \mathrm{ET}_{\mathrm{RBGE}}+\mathrm{ET}_{\mathrm{KVMX}}+\mathrm{ET}_{\mathrm{KVGO}}+\mathrm{ET}_{\mathrm{KVGE}}+ \\
& \mathrm{ET}_{\mathrm{MXGO}}+\mathrm{ET}_{\mathrm{MXGE}}+\mathrm{ET}_{\mathrm{GOGE}},
\end{aligned}
$$

em que $\mathrm{PE}_{\mathrm{w}}$ representa a porcentagem da energia total do modo horizontal w em relação a todos os modos, incluindo as interações entre eles, contida na categoria de modos verticais considerada. Ewn, ETsoma e ETint são, respectivamente, as energias totais de cada modo horizontal w, a soma de todos os modos horizontais e as interações entre os pares de modos horizontais indexados, para um dado modo vertical n. Integrados globalmente, os produtos cruzados se anulam (ortogonalidade das Funções de Hough), entretanto, localmente, estes produtos podem ser interpretados como interferência construtiva (valores positivos) ou destrutiva (valores negativos) de energia, resultante da interação entre os modos que produzem o produto. $\mathrm{O}$ limiar $\mathrm{M}$ da somatória no denominador de (3) denota o número de modos verticais considerados, neste caso, $\mathrm{M}=12$ (14) para 28 (42) níveis verticais.

Estes cálculos são realizados para a região da América do Sul, enfatizando-se a região média de atuação do composto dos episódios de ZCAS, conforme mostrado na Figura 2. O segmento $\mathrm{AB}$ indica o eixo médio da confluência do campo composto de vento horizontal em $850 \mathrm{hPa}$ (não apresentado), que coincide com a banda de nebulosidade associada ao composto de ZCAS. A técnica utilizada para composição dos episódios de ZCAS é descrita na parte I deste artigo (MB08).

\subsection{Experimentos}

O desempenho do modelo global do CPTEC em reproduzir a partição de energia observada em modos verticais e horizontais é avaliado a partir de seis experimentos, utilizandose além das diferentes resoluções (T126L28 e T170L42), três diferentes esquemas de convecção profunda: KUO (Kuo, 1965 e Anthes, 1977), utilizado operacionalmente no MCGA-CPTEC 


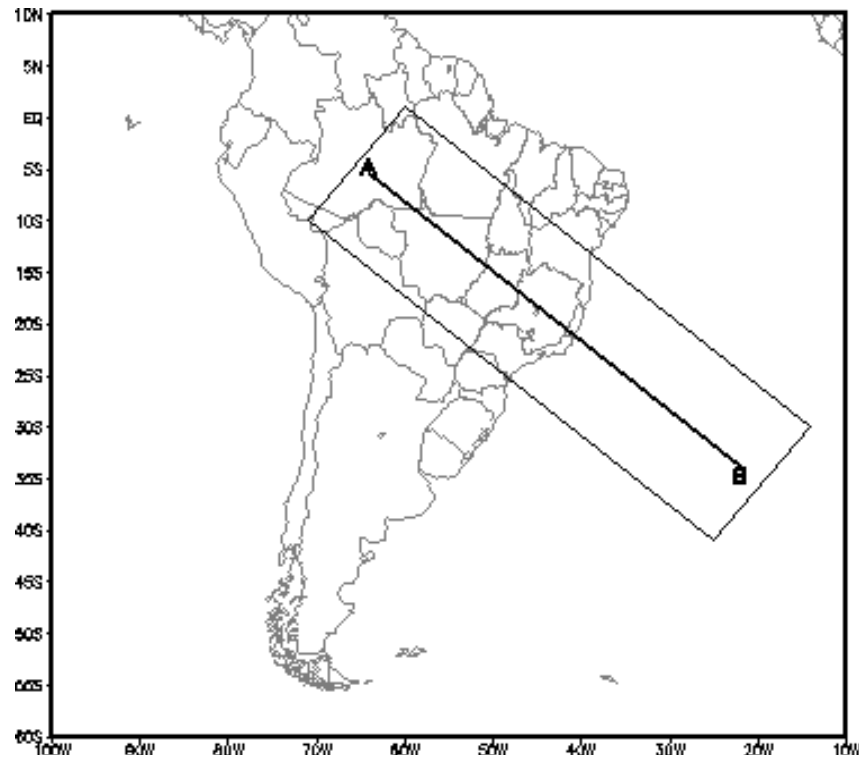

Figura 2 - Região média de atuação do composto de ZCAS, definida para a partição modal vertical de energia sobre a América do Sul.

e, Relaxed Arakawa-Schubert - RAS (Moorthi e Suarez, 1992) e GRELL (Grell et al., 1994), que são os esquemas opcionais do MCGA-CPTEC. Estes experimentos são resumidos na Tabela 1.

Os cálculos da partição de energia entre os modos normais verticais e horizontais são realizados para as previsões de 24 horas, válidas para o período de cada episódio de ZCAS descrito na seção 2.3. É importante salientar que o prazo de 24 horas para as previsões foi selecionado pelo fato de que os erros do modelo aparecem já nas primeiras horas de integração. Em alguns casos, um prazo de 6 horas é suficiente para identificar tais erros (Figueroa, 2008 - Comunicação Pessoal). As previsões são geradas a partir das rodadas do MCGA-CPTEC com resoluções T126L28 e T170L42, partindo das condições iniciais do NCEP correspondentes aos episódios selecionados.

\section{RESULTADOS E DISCUSSÕES}

Nesta parte do trabalho (parte de modelagem), os resultados são avaliados sob três aspectos: (i) capacidade do modelo em reproduzir a energética observada; (ii) impacto do aumento da resolução espacial do modelo e (iii) influência da utilização de diferentes esquemas de convecção na energética modal. A idéia não é verificar a capacidade do modelo em simular a ZCAS, mas analisar se o modelo global do CPTEC é capaz de reproduzir a partição modal de energia observada, e se diferentes resoluções do modelo e diferentes esquemas de convecção profunda influenciam no desempenho do modelo em simular a energética modal da ZCAS. Os resultados obtidos na análise observacional (MB08) serão considerados como referência ou controle para avaliação do desempenho do modelo e são resumidos na Tabela 2.

De acordo com o definido na parte observacional, a análise dos resultados é realizada para três categorias de modos verticais: (I) $n=1$ a 3, com $\mathrm{Hn}$ acima de $600 \mathrm{~m}$; (II) $\mathrm{n}=4$ a 7, com Hn entre 100 e $600 \mathrm{~m}$; e (III) n=8 a 12 (14, para 42 níveis), com Hn entre 10 e $100 \mathrm{~m}$.

\subsection{Impacto da Resolução do Modelo e Diferentes Parametrizações de Convecção}

\subsubsection{Partição de Energia entre os Modos Verticais}

Os campos de porcentagem de energia para os experimentos de modelagem, utilizando os esquemas de convecção KUO, RAS e GRELL, são apresentados nas Figuras 3-5. Em geral, os resultados são, quantitativa e qualitativamente, bastante semelhantes aos dos experimentos de controle (Figura 8 de MB08), para ambas as resoluções. O modelo com esquema GRELL apresenta uma melhor definição dos padrões espaciais observados, especialmente na região da ZCAS. Entretanto, o

Tabela 1- Descrição dos Experimentos de Modelagem

\begin{tabular}{|c|c|c|}
\hline Experimentos & Resolução & Esquema de Convecção \\
\hline Experimento 1 (EXP1) & Modelo T126L28 & KUO \\
Experimento 2 (EXP2) & Modelo T170L42 & \\
\hline Experimento 3 (EXP3) & Modelo T126L28 & RAS \\
Experimento 4 (EXP4) & Modelo T 170L42 & \\
\hline Experimento 5 (EXP5) & Modelo T126L28 & GRELL \\
Experimento 6 (EXP6) & Modelo T170L42 & \\
\hline
\end{tabular}


Tabela 2 - Partição Horizontal de Energia - Resultados Observacionais

\begin{tabular}{|c|c|c|}
\hline Modos Verticais & Auto-Interaçōes & Interaçóes Horizontais \\
\hline $\begin{array}{l}\text { Categoria I: } \\
\text { Modos } 1 \text { a } 3\end{array}$ & $\begin{array}{l}\text { Rossby: em latitudes altas } \\
\text { Kelvin: ZCAS e próximo ao equador. } \\
\text { Obs.: Resposta em latitudes altas do modo } \\
\text { Rossby ao aquecimento equatorial, geradas } \\
\text { pelo modo externo. As maiores } \\
\text { contribuiçóes do modo Kelvin ocorrem em } \\
\text { regiōes convectivas. }\end{array}$ & $\begin{array}{l}\text { Rossby-Kelvin } \\
\text { Contribuiçôes secundárias na } \\
\text { região da ZCAS: } \\
\text { Rossby-Misto } \\
\text { Kelvin-Misto } \\
\text { Kelvin-Grav. Oeste } \\
\text { Kelvin-Grav. Leste }\end{array}$ \\
\hline $\begin{array}{l}\text { Categoria II: } \\
\text { Modos } 4 \text { a } 7\end{array}$ & $\begin{array}{l}\text { Rossby: América do Sul central, incluindo } \\
\text { a ZCAS } \\
\text { Kelvin: região equatorial } \\
\text { Obs.: Máximas porcentagens de energia } \\
\text { confinadas equatorialmente, com valores } \\
\text { acima de } 100 \% \text {, indicando interação entre } \\
\text { os modos horizontais. }\end{array}$ & $\begin{array}{l}\text { Rossby-Kelvin } \\
\text { Contribuiçóes secundárias nla } \\
\text { região da ZCAS: } \\
\text { Rossby-Misto } \\
\text { Rossby-Grav. Oeste } \\
\text { Rossby-Grav. Leste }\end{array}$ \\
\hline $\begin{array}{l}\text { Categoria III: } \\
\text { Modos } 8 \text { a } 12 \text { (14) }\end{array}$ & $\begin{array}{l}\text { Rossby: em latitudes médias e altas, } \\
\text { incluindo a ZCAS } \\
\text { Kelvin: região equatorial } \\
\text { Obs.: Contribuições significativas e } \\
\text { energia do modo Rossby, com padrôes } \\
\text { bem definidos na região da ZCAS. }\end{array}$ & Rossby-Kelvin \\
\hline
\end{tabular}




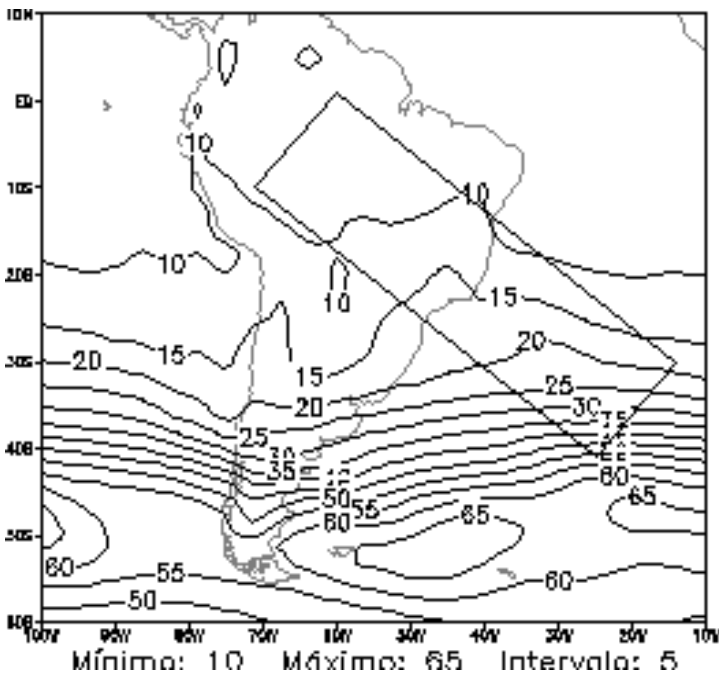

(a)

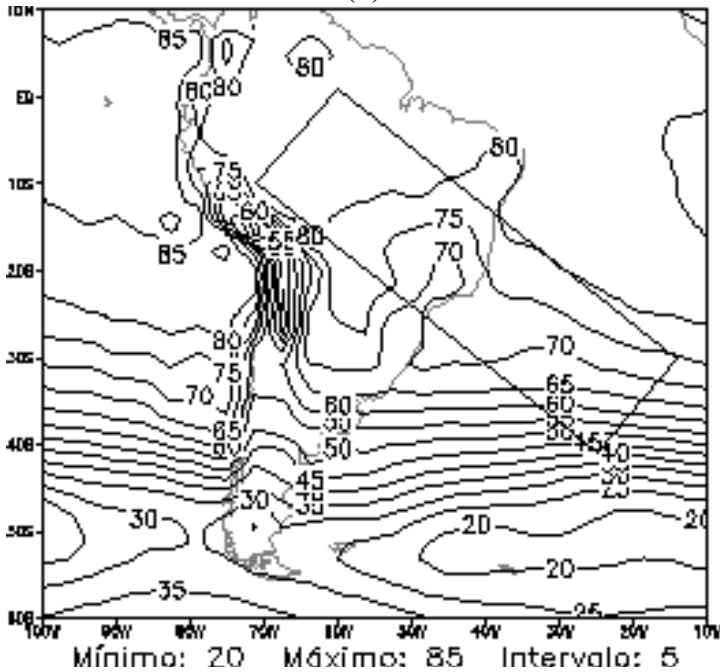

(c)

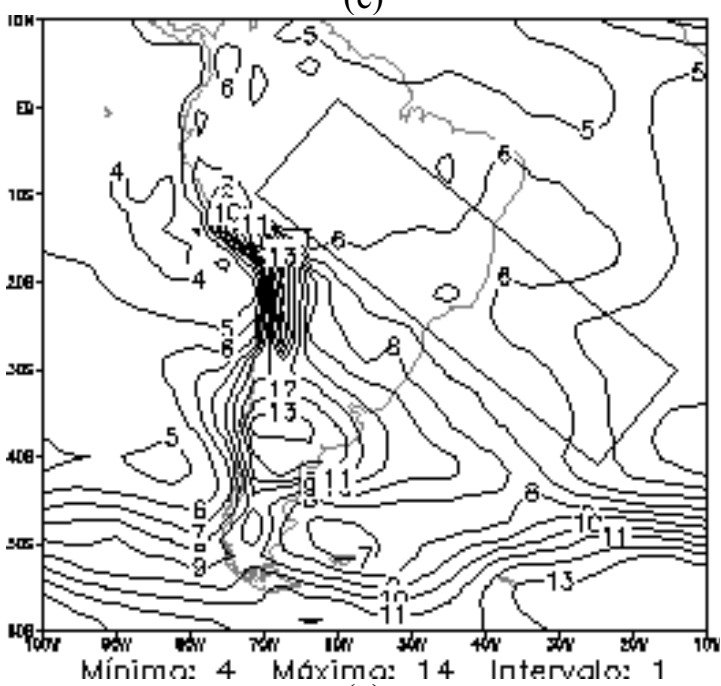

(e)

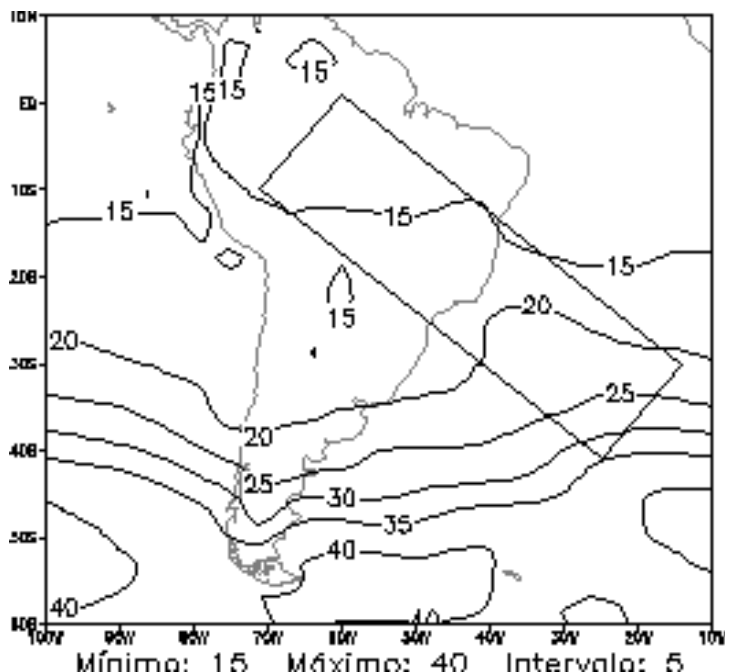

(b)

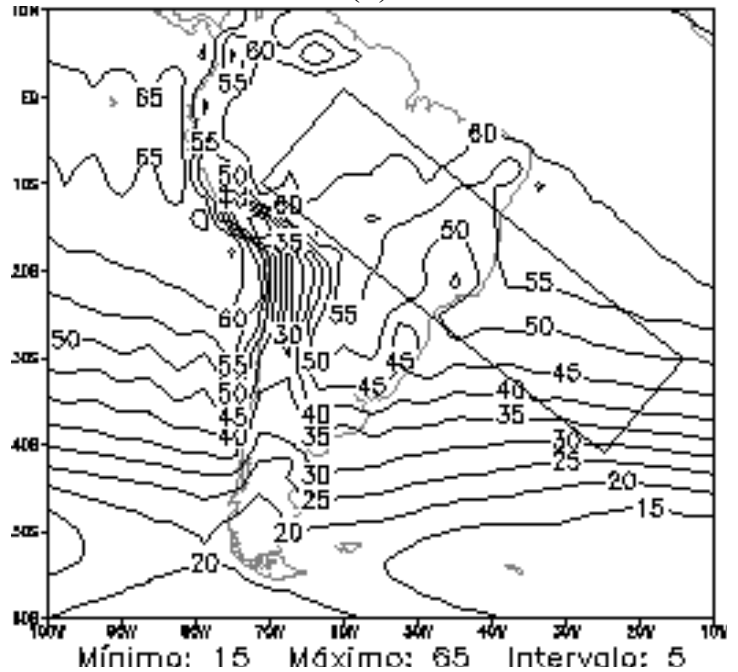

(d)

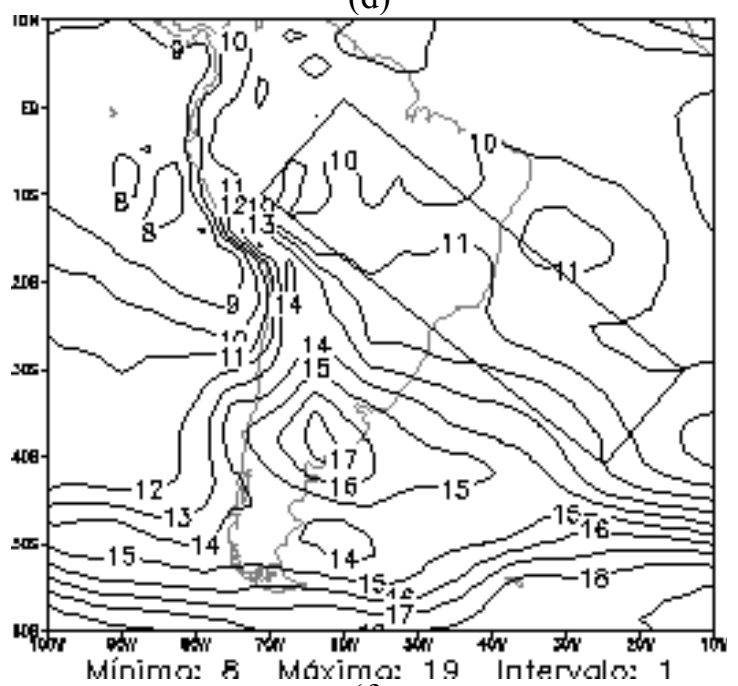

(f)

Figura 3 - Energia Total (\%), MCGA-CPTEC - KUO, média das previsões de 24h, T126L28 (a esquerda) e T170L42 (a direita): (a) e (b) modos verticais 1 a 3; (c) e (d) modos verticais 4 a 7; e (e) e (f) modos verticais 8 a 12 (8 a 14, para T170L42). 


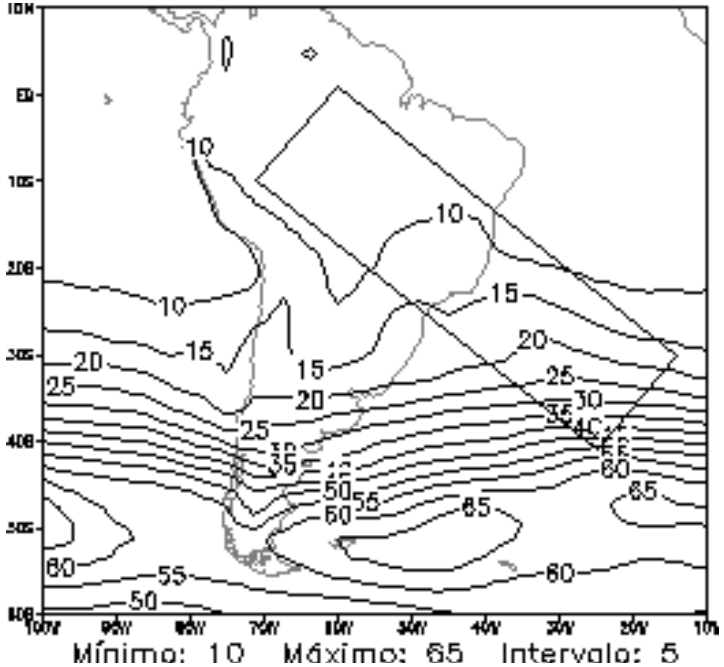

(a)

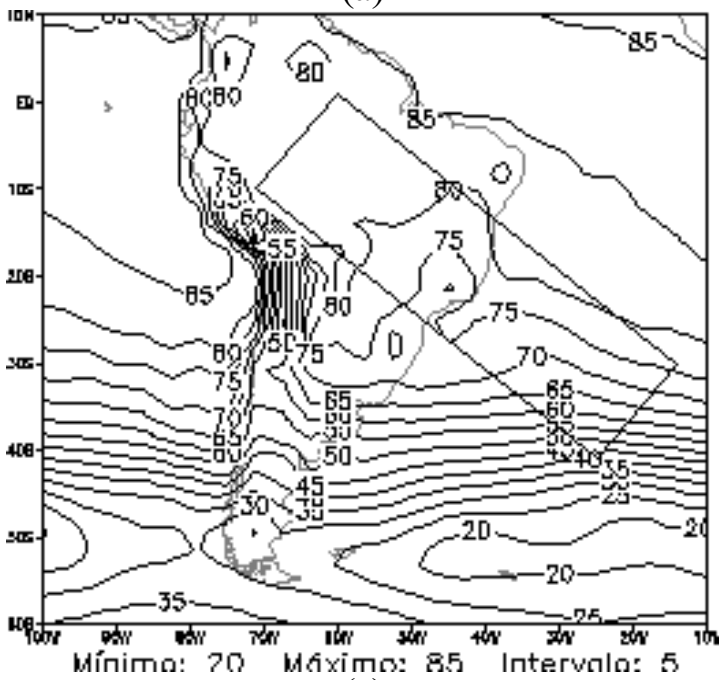

(c)

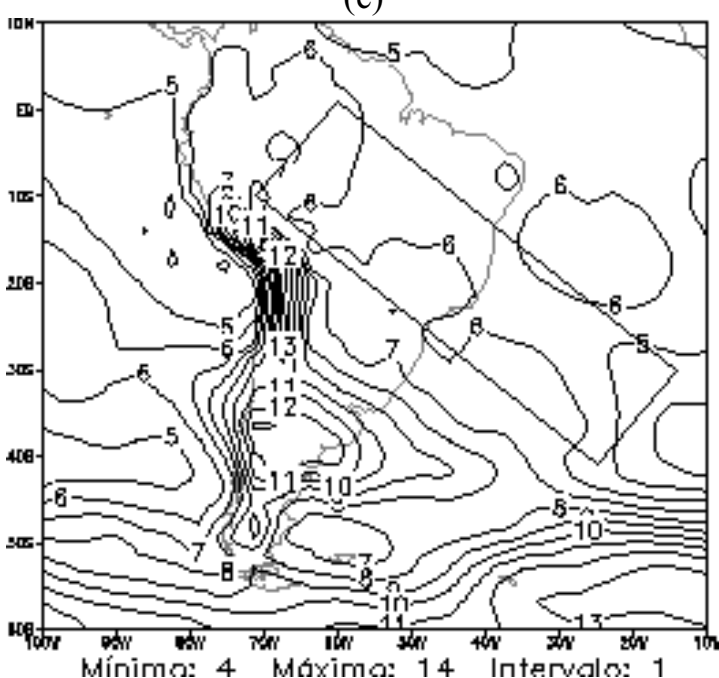

(e)

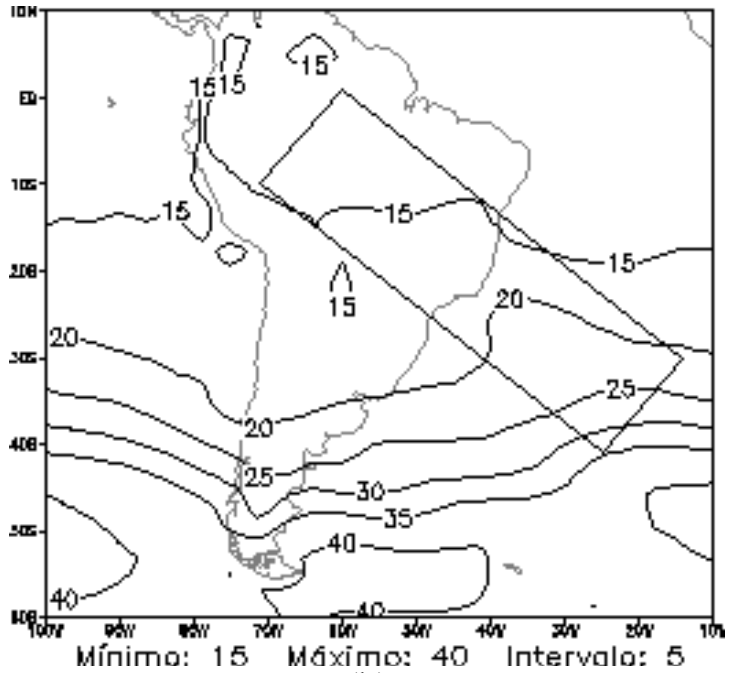

(b)

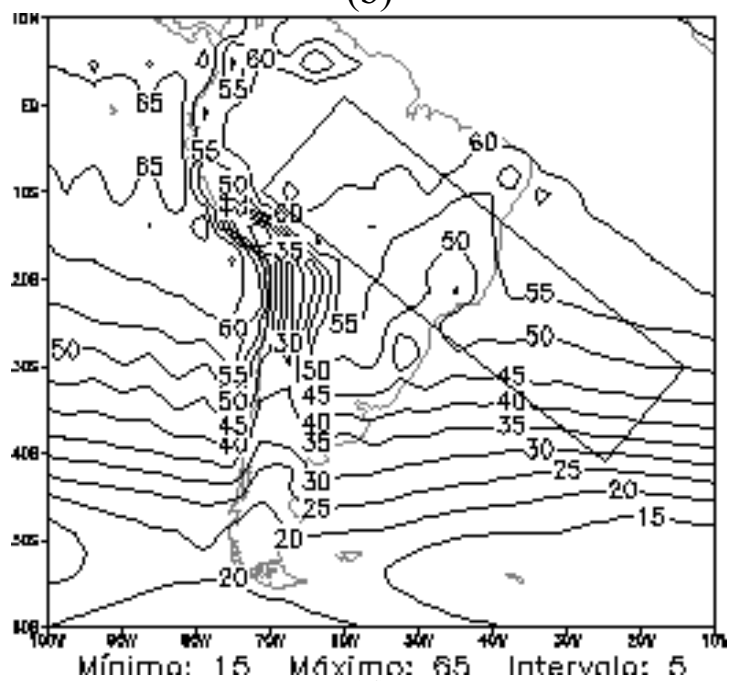

(d)

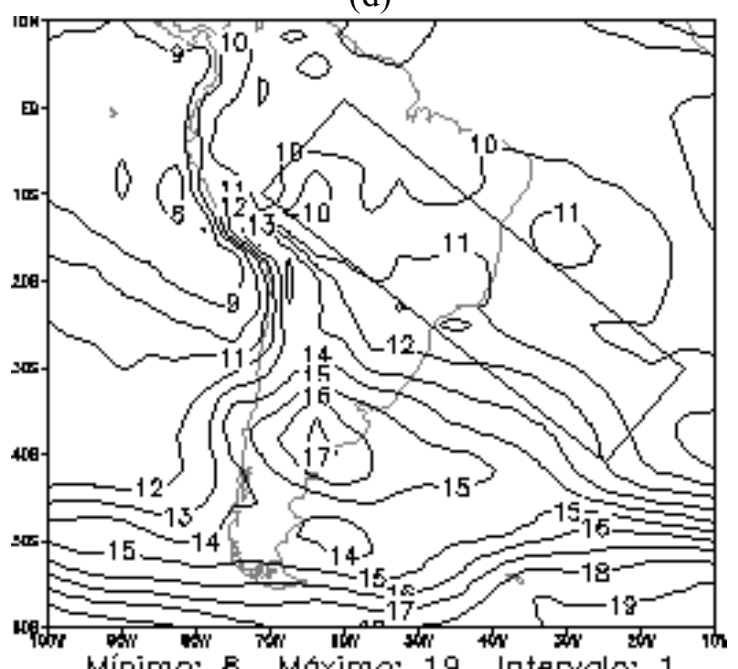

(f)

Figura 4 - Energia Total (\%), MCGA-CPTEC - RAS, média das previsões de 24h, T126L28 (a esquerda) e T170L42 (a direita): (a) e (b) modos verticais 1 a 3; (c) e (d) modos verticais 4 a 7; e (e) e (f) modos verticais 8 a 12 (8 a 14, para T170L42). 


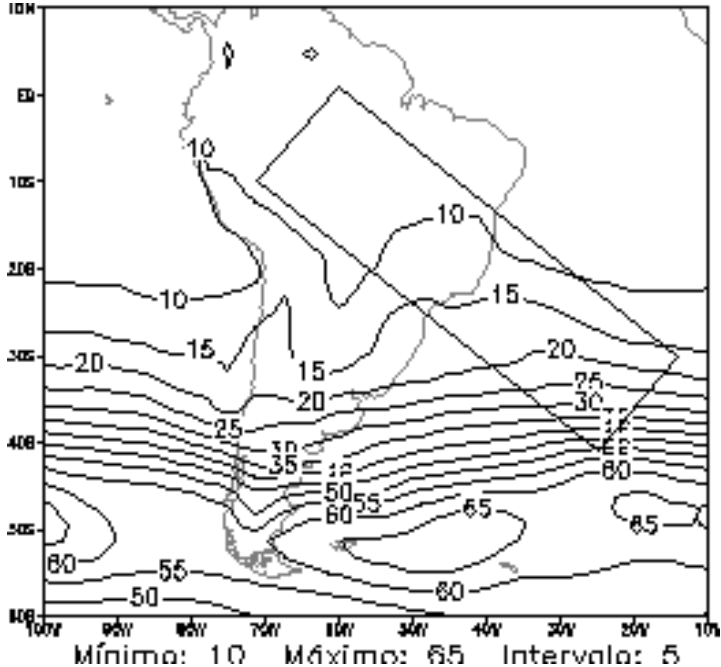

(a)

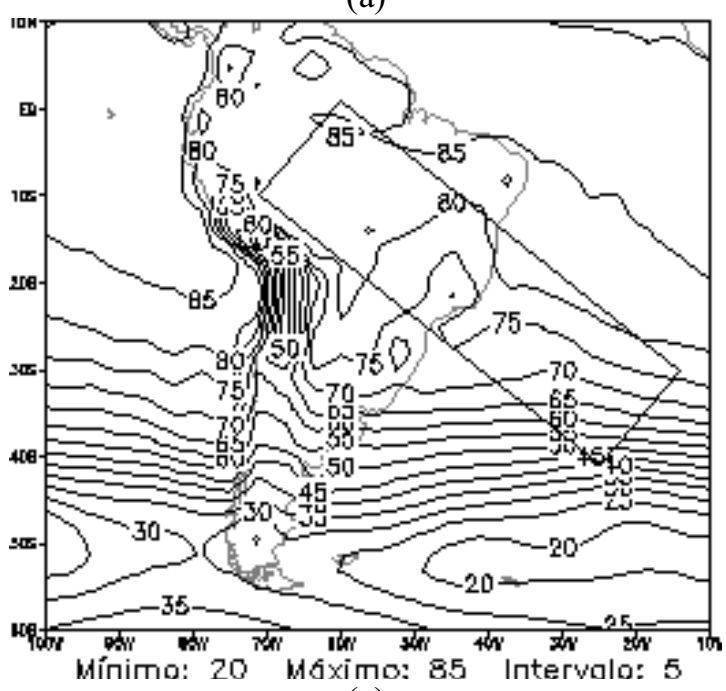

(c)

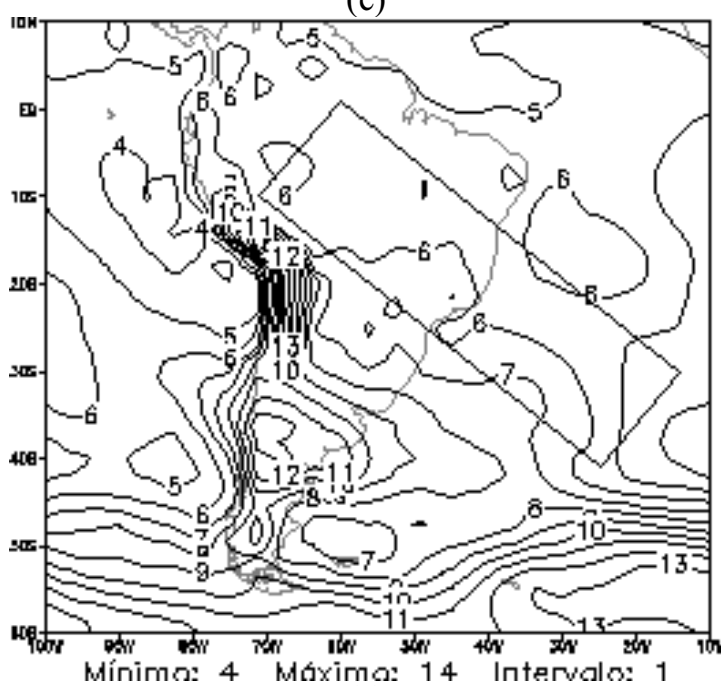

(e)

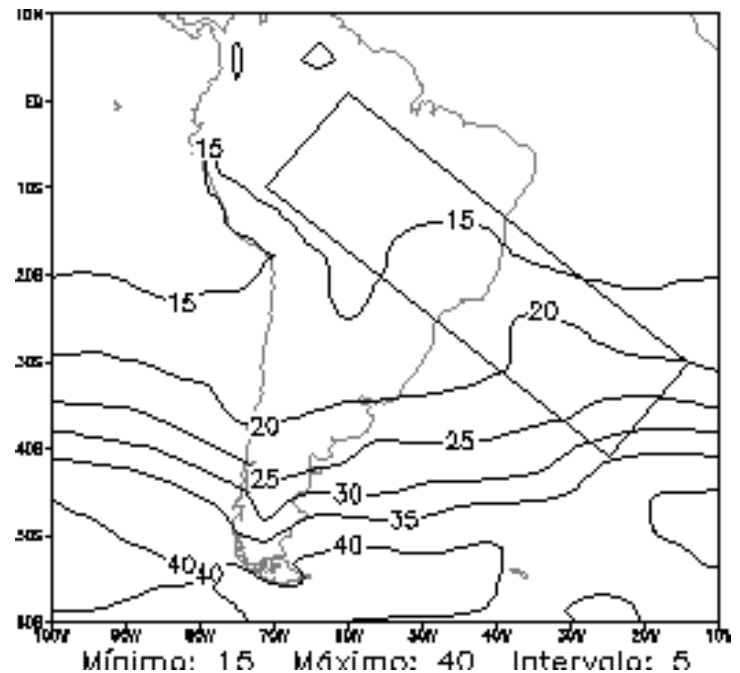

(b)

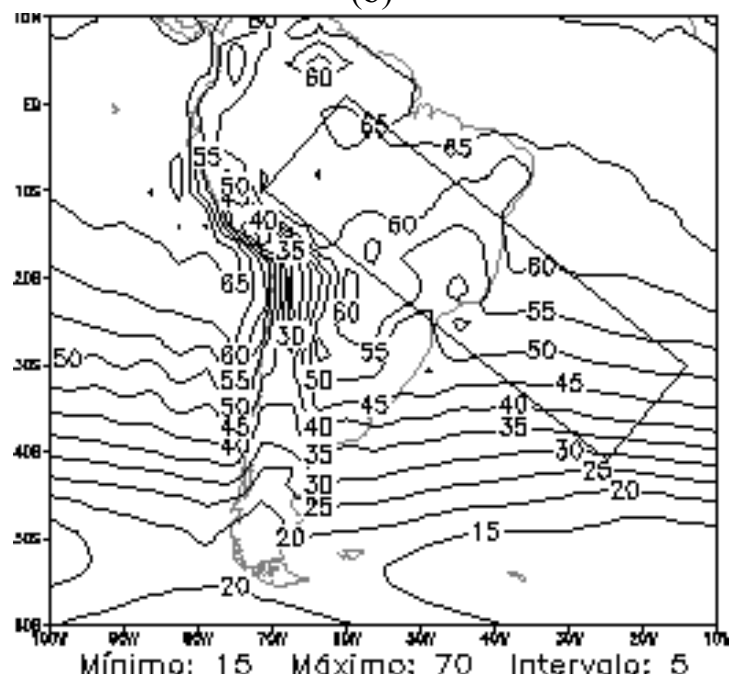

(d)

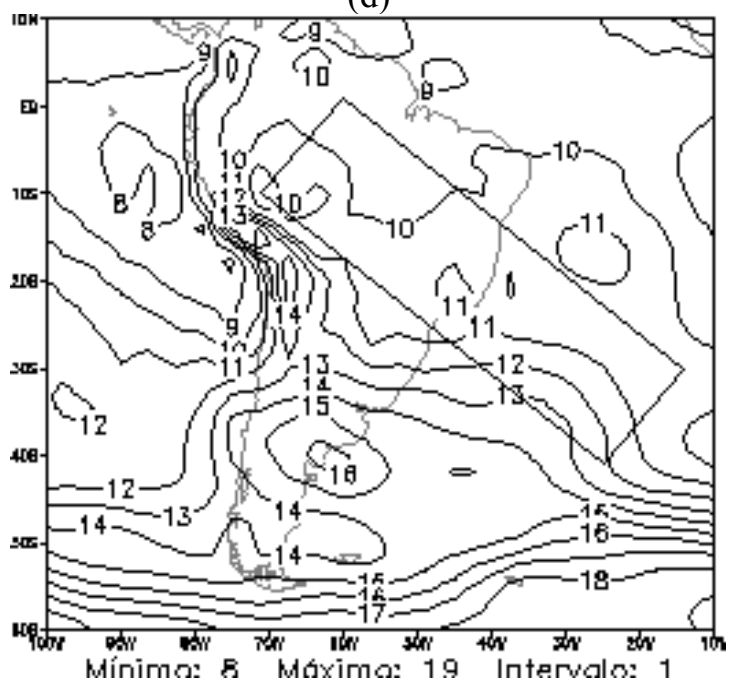

(f)

Figura 5 - Energia Total (\%), MCGA-CPTEC - GRELL, média das previsões de 24h, T126L28 (a esquerda) e T170L42 (a direita): (a) e (b) modos verticais 1 a 3; (c) e (d) modos verticais 4 a 7; e (e) e (f) modos verticais 8 a 12 (8 a 14, para T170L42). 
comportamento do modelo com os três esquemas de convecção profunda não apresentou diferenças significativas, com todos os esquemas simulando satisfatoriamente o padrão e os núcleos de máxima porcentagem de energia, e indicando resultados similares entre si. Isto sugere que, para estes experimentos, o esquema de convecção utilizado no modelo não tem grandes impactos na energética modal. Impactos maiores são observados quando considerado o aumento da resolução vertical.

A comparação dos resultados das Figuras 3-5 com os campos de precipitação total apresentados na Figura 6 indica que os núcleos de máxima porcentagem de energia estão em concordância com as áreas convectivamente ativas e com máximos de precipitação, especialmente para os modos verticais da categoria II. Os modos verticais 4 e 5 são aqueles mais excitados pelo aquecimento convectivo, na região tropical (Puri, 1983). Destaca-se ainda da Figura 6, que o Modelo Global do CPTEC T170L42 utilizando o esquema GRELL apresenta uma melhor previsão do campo de precipitação, especialmente sobre o continente.

Na Figura 7 são mostradas as fontes e sumidouros de calor, previstas pelo MCGA-CPTEC utilizando os esquemas de convecção profunda KUO, RAS e GRELL. Em geral, o padrão das fontes e sumidouros de calor é bem previsto pelo modelo global para os três esquemas de convecção profunda, entretanto, quando comparado aos resultados das análises T126L28 e T170L42 (Figura 9 de MB08) nota-se que há uma sub-estimativa da intensidade das fontes de calor, especialmente sobre a parte oceânica do composto de ZCAS e sobre a Zona de Convergência Intertropical (ZCIT), e superestimativa de alguns núcleos de fontes e sumidouros de calor sobre o continente Sul-Americano.

\subsubsection{Partição de Energia entre os Modos Horizontais}

De um modo geral, a análise subjetiva dos resultados desta subseção indica que, em ambas as resoluções, o modelo reproduz os padrões observados na partição de energia entre os modos horizontais, independente do esquema de convecção profunda utilizado, para todas as categorias de modos verticais. Para a resolução T126L28, uma análise global dos resultados mostra que os esquemas KUO e RAS tendem a apresentar resultados ligeiramente melhores, embora o esquema GRELL mostre um maior detalhamento de alguns padrões, regionalmente. Já a combinação do modelo com resolução espacial mais alta (T170L42) e o esquema GRELL tendem a apresentar uma pequena melhoria nos resultados, com alguns padrões regionais reproduzidos mais precisamente, principalmente para as categorias I e III. Entretanto, o modelo com o esquema RAS parece reproduzir melhor os padrões globais, verificados nos experimentos de controle.
Devido à grande quantidade de informações obtidas nesta análise, são mostradas a seguir apenas as figuras referentes aos resultados da categoria II de modos verticais, a qual detém a maior porcentagem de energia na região de interesse do estudo. Para as categorias I e III, os resultados são discutidos e sintetizados de forma objetiva nas Tabelas 3 e 4 .

Para todos os esquemas de convecção e resoluções espaciais, a média das previsões de $24 \mathrm{~h}$ tende a superestimar as porcentagens de energia total para a soma de todas as autointerações da categoria I, em latitudes médias e altas. Embora os padrões previstos sejam muito similares entre si, o modelo com esquema KUO apresenta melhor desempenho na região equatorial, tanto para T126L28 como para T170L42. Em latitudes médias, o modelo com o esquema GRELL e resolução T170L42 reproduz os padrões observados com um maior detalhamento. Para o modo Rossby, o modelo T170L42, com o esquema GRELL tende a suavizar o campo de porcentagem de energia total; enquanto os esquemas KUO e RAS conseguem prever bem os detalhes dos padrões observados na região equatorial. Para o modo Kelvin, observa-se um bom desempenho do modelo tanto em prever os padrões espaciais, como os valores de porcentagem de energia obtidos nos experimentos de controle (MB08). Na região equatorial, o modelo com o esquema KUO define melhor o padrão espacial para a resolução T126L28; as previsões com os esquemas RAS e GRELL foram muito similares, sendo que este último tende a realçar os detalhes regionais do campo. Para a resolução T170L42, os modelos reproduziram bem o padrão espacial, notando-se um melhor desempenho do modelo com o esquema RAS, comparado ao modelo de baixa resolução; o esquema GRELL descreve melhor o padrão espacial, porém subestima as porcentagens de energia em algumas regiões.

As Figuras 8-10 mostram, respectivamente, as porcentagens de energia total da soma de todas as autointerações (Soma), modo Rossby e modo Kelvin, previstas pelo modelo global com resoluções T126L28 e T170L42, para os modos verticais 4 a 7. No caso da Soma (Figura 8), os resultados do modelo para a região da ZCAS foram ligeiramente melhores para a resolução T170L42. Apesar da similaridade entre os padrões previstos pelos três esquemas, em ambas resoluções o esquema RAS parece representar melhor os padrões observados (Figura 11a,b de MB08) embora superestime os núcleos de máxima porcentagem de energia na região equatorial no modelo T126L28.

Para o modo Rossby (Figura 9), nota-se que, quantitativamente, o modelo T126L28 com o esquema KUO consegue prever bem os núcleos de máxima porcentagem de energia na região equatorial. Em latitudes médias e altas, a comparação entre os modelos mostra que os padrões previstos, utilizando os três esquemas de convecção, são bastante semelhantes entre si. Em relação a análise T126L28 observados 

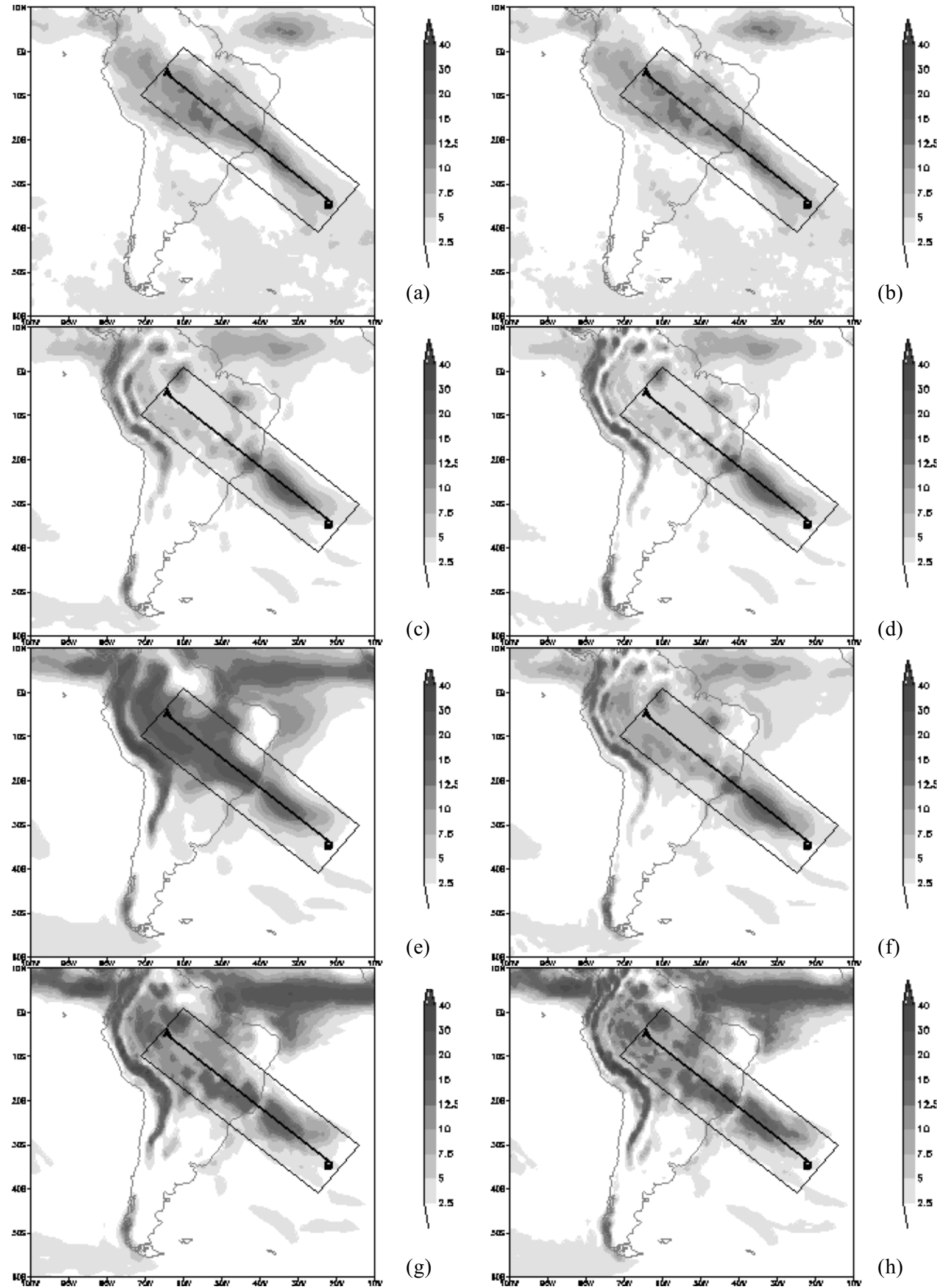

(a)

(c)
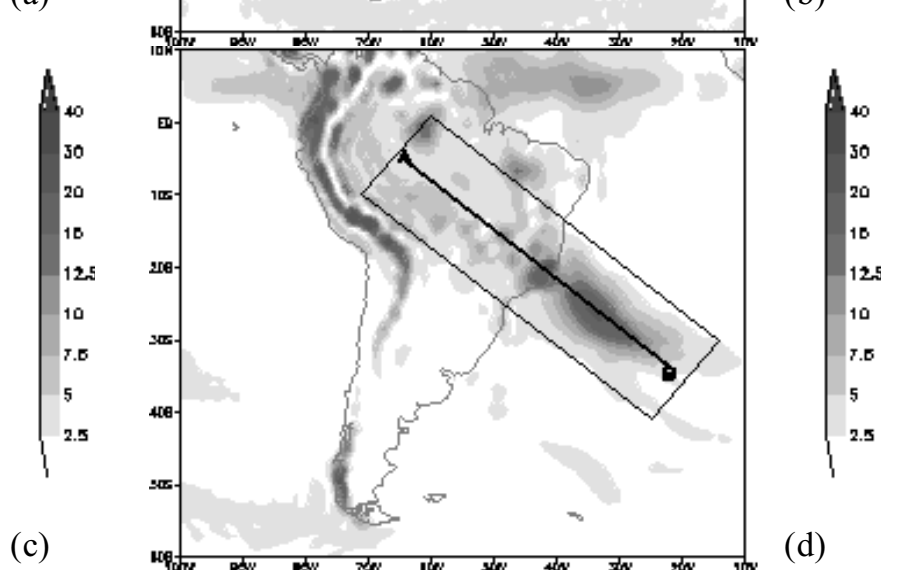

(b)

(e)
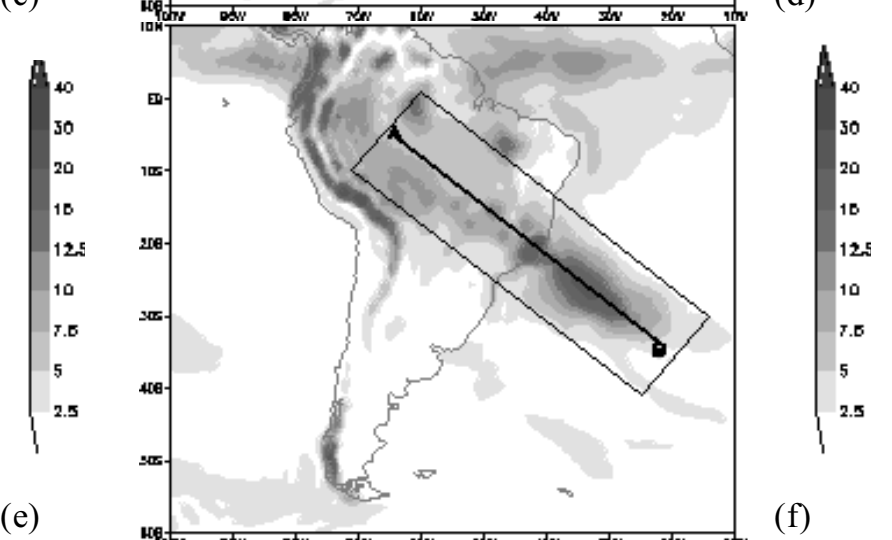

(g)
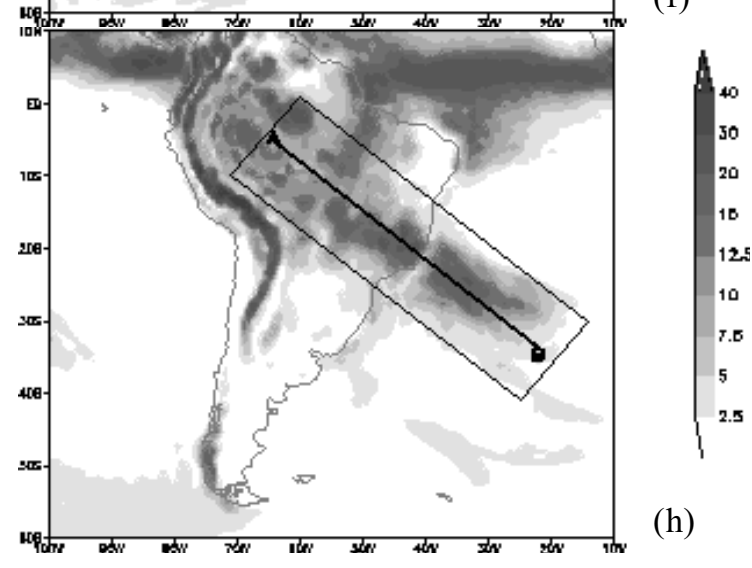

Figura 6 - Precipitação Total (mm/dia), para o composto de ZCAS: (a) e (b) dados GPCP, (c)-(h) MCGA-CPTEC - KUO, RAS e GRELL respectivamente, para as resoluções T126L28 (a esquerda) e T170L42 (a direita). 
(Figura 11c de MB08), nota-se que os modelos tendem a suavizar as isolinhas na região da ZCAS. Na resolução T170L42, os modelos apresentaram resultados muito semelhantes, reproduzindo bem os padrões observados. Na Figura 10, as auto-interações do modo Kelvin mostram que os modelos têm um bom desempenho em ambas as resoluções, com todos os esquemas de convecção representando muito bem os padrões de controle. Resultados ligeiramente melhores são observados para o esquema KUO (RAS) na resolução T126L28 (T170L42).
A análise da partição de energia entre os modos horizontais para a categoria III (Figuras não mostradas) indica que, em geral, o modelo com o esquema GRELL é o que apresenta um melhor desempenho nesta categoria, prevendo com maior detalhamento os padrões em torno do equador e sobre a região da ZCAS. Para a soma de todas as auto-interações, o modelo com o esquema KUO tende a subestimar os valores na região equatorial, enquanto o modelo RAS superestima os valores nesta região. No caso dos modos Rossby e Kelvin, não
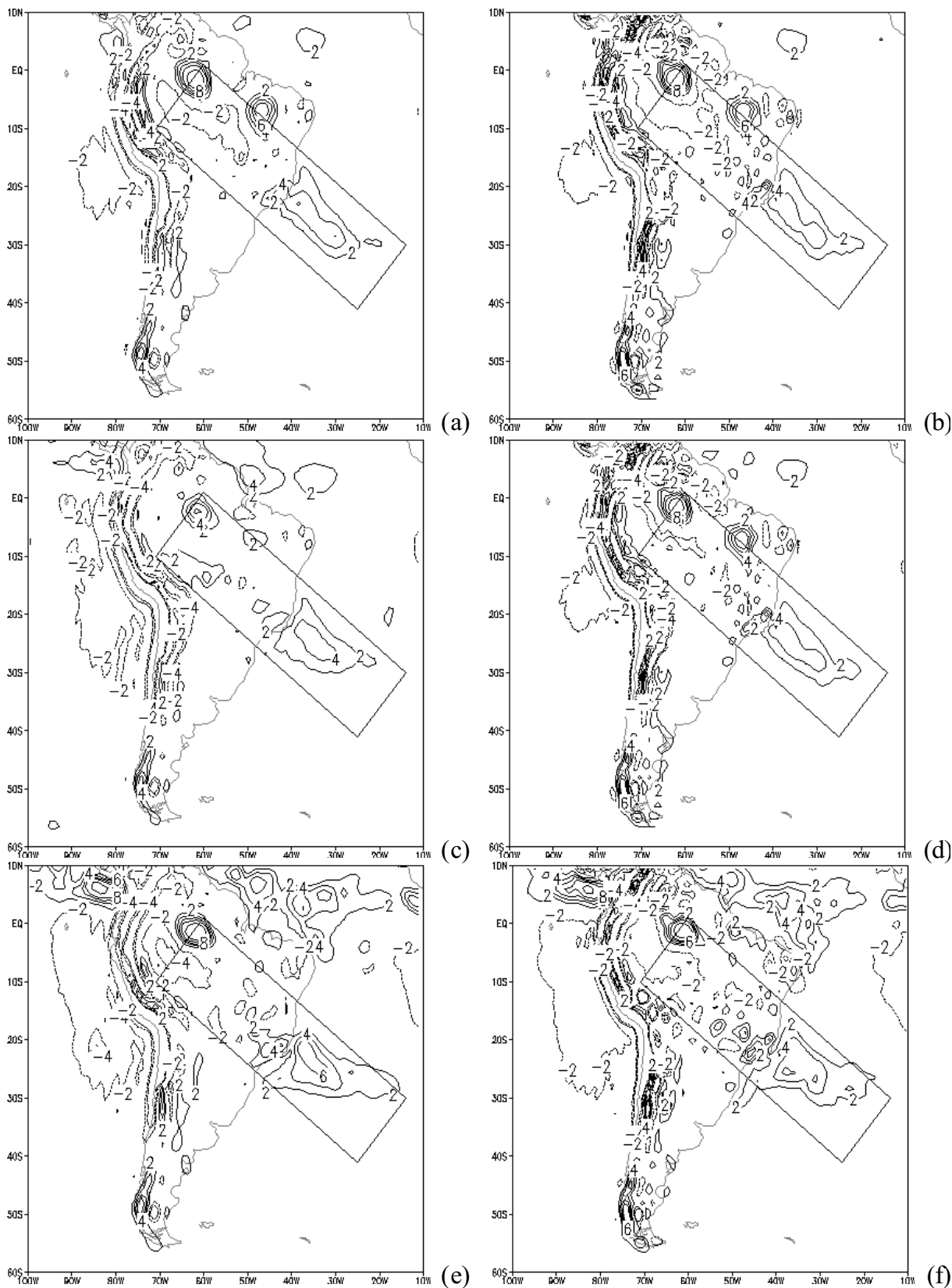

Figura 7- Fontes e sumidouros de calor (K/dia) para o composto de ZCAS, média entre os níveis 700 hPa e 250 hPa, previsão de 24 horas, MCGACPTEC -T126L28 (a esquerda) e T170L42 (a direita), respectivamente: (a) e (b) KUO, (c) e (d) RAS, (e) e (f) GRELL. 
há diferenças significativas entre os padrões previstos pelos três esquemas de convecção e as análises T126L28 e T170L42.

A soma das interações entre todos os modos horizontais e os produtos cruzados entre modos horizontais para os modos verticais 1 a 3 (categoria I) indicam um melhor desempenho do modelo com o esquema KUO para a resolução T126L28, e com o esquema RAS para a resolução T170L42, em comparação aos resultados obtidos para os experimentos de controle. Os principais padrões obtidos na análise diagnóstica da energética

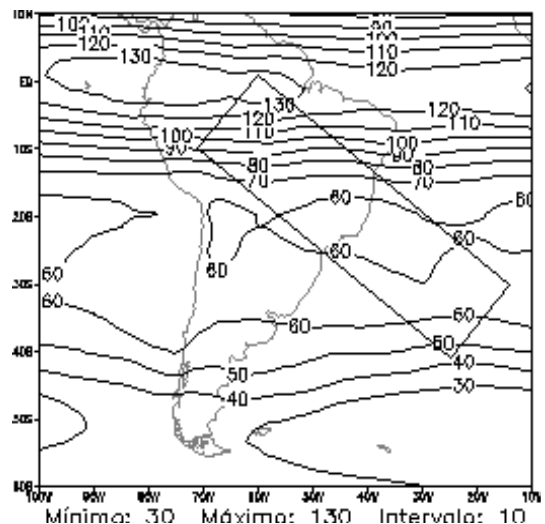

(a)

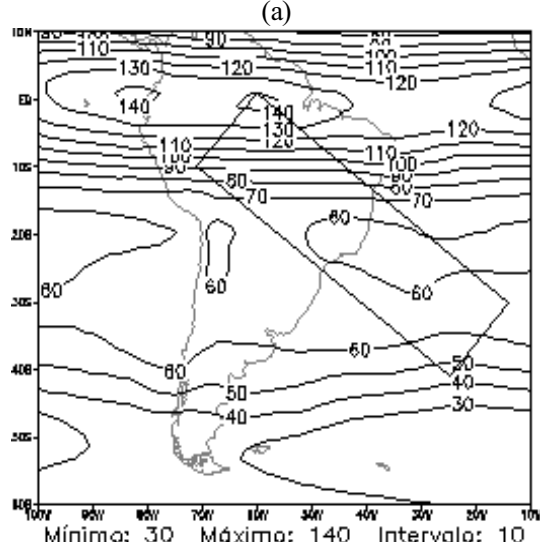

(c)

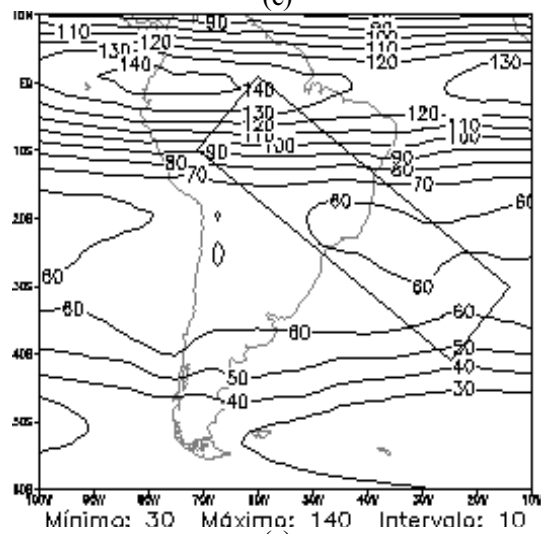

(e) modal apresentada em MB08 foram qualitativamente bem representados em todas as configurações do modelo.

As Figuras 11 e 12 representam as principais interações entre os modos horizontais para os modos verticais 4 a 7 , previstas pelo MCGA-CPTEC com resoluções T126L28 e T170L42. A soma de todas as interações (Resíduo) para esta categoria indica que os modelos T126L28 e T170L42 conseguem reproduzir bem os padrões globais observados nos experimentos de controle (Figura 14 de MB08), com previsões

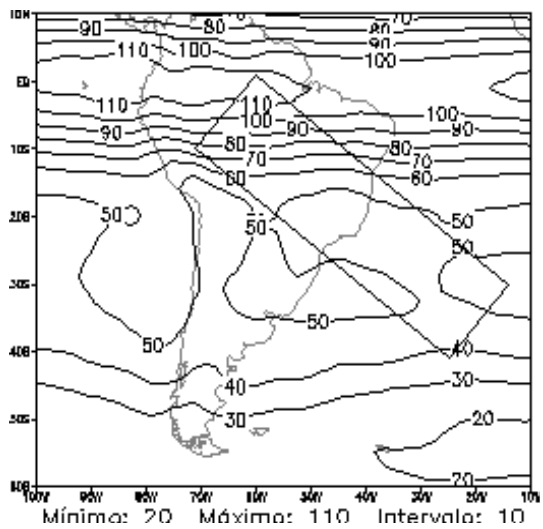

(b)

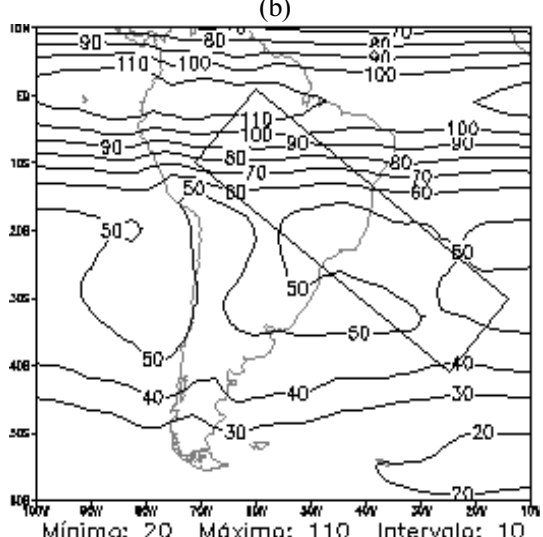

(d)

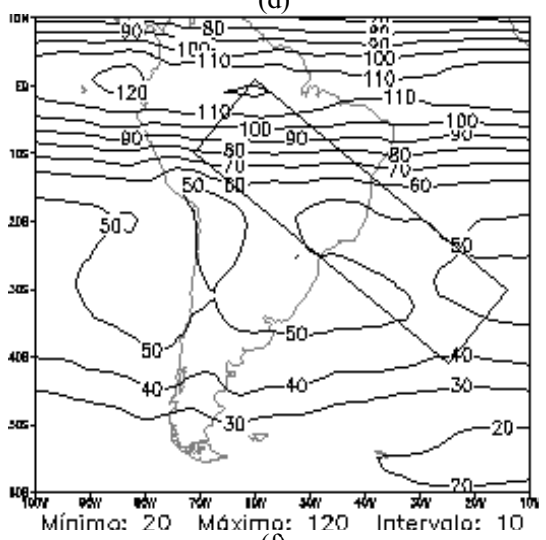

(f)

Figura 8 - Energia Total (\%) do modo horizontal Soma para os modos verticais 4 a 7, média das previsões de 24h, MCGA-CPTEC - T126L28 (a esquerda) e T170L42 (a direita): (a) e (b) KUO; (c) e (d) RAS; (e) e (f) GRELL. 
muito semelhantes para os três esquemas de convecção. Para ambas as resoluções, nota-se que o modelo com o esquema RAS apresenta resultados ligeiramente melhores, embora o esquema GRELL reproduza os padrões regionais com maiores detalhes (Figura 11). Para as interações entre os modos Rossby e Kelvin (Figura 12), as previsões obtidas com os esquemas de convecção profunda KUO, RAS e GRELL não apresentaram diferenças significativas entre si. Em geral, os valores e os padrões espaciais dos núcleos de máxima interferência construtiva/destrutiva

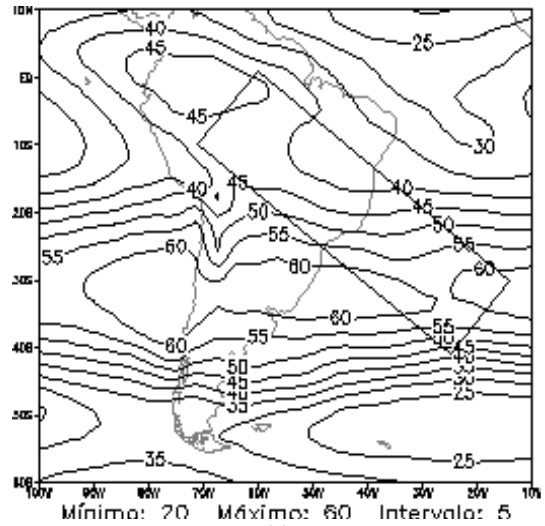

(a)

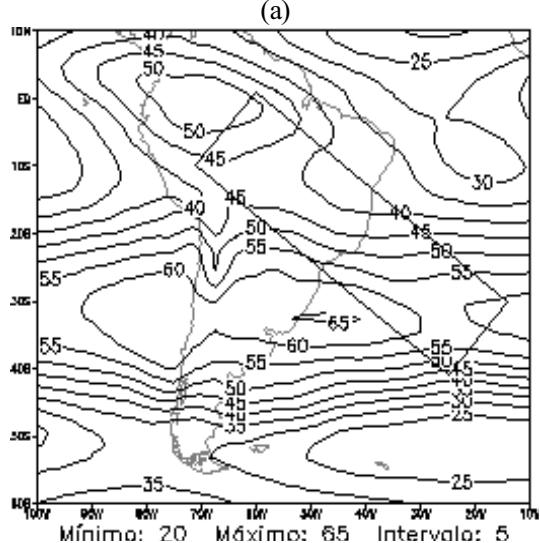

(c)

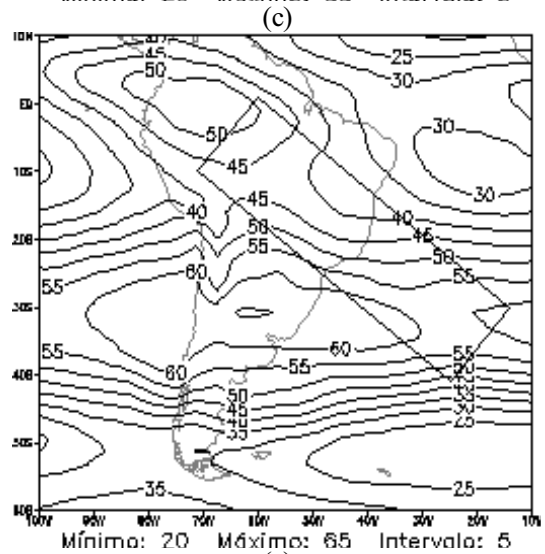

(e) de energia foram bem previstos, verificando-se um melhor desempenho do modelo com os esquemas KUO e RAS para as resoluções T126L28 e T170L42, respectivamente. Para a resolução T170L42, o modelo com o esquema GRELL tende a superestimar os núcleos de máxima interação.

O desempenho do modelo para as previsões das interações entre os demais pares de modos horizontais, para a categoria II de modos verticais, são resumidos nas Tabelas 3 e 4 . Como mostrado nos resultados anteriores, há uma boa

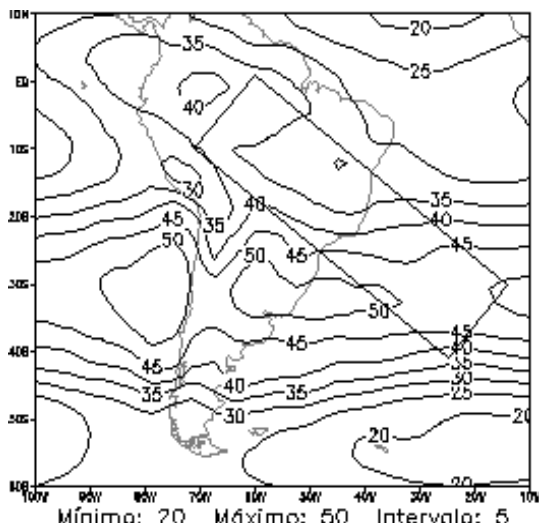

(b)

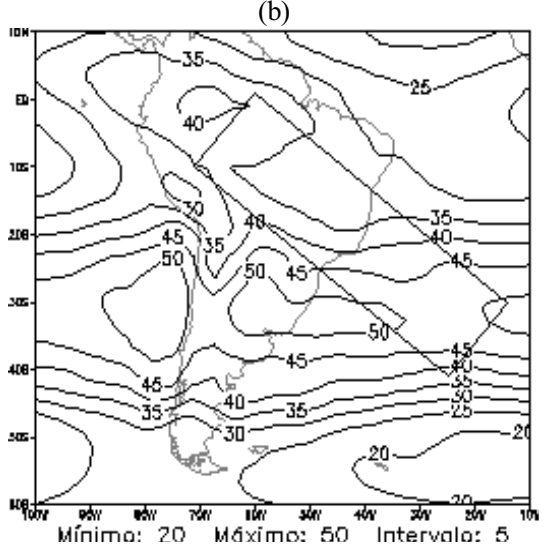

(d)

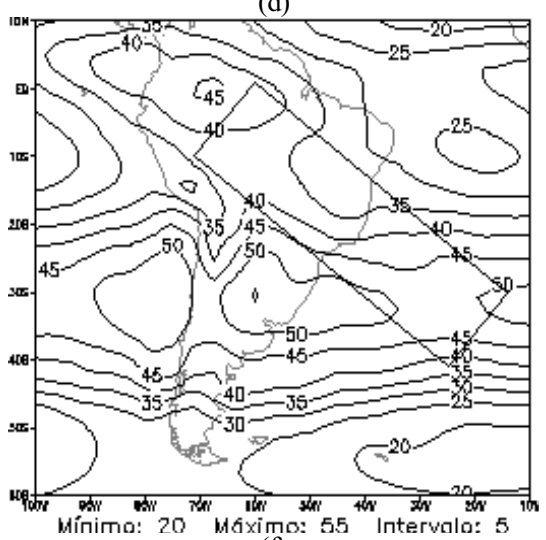

(f)

Figura 9 - Energia Total (\%) do modo horizontal Rossby para os modos verticais 4 a 7, média das previsões de 24h, MCGA-CPTEC - T126L28 (a esquerda) e T170L42 (a direita): (a) e (b) KUO; (c) e (d) RAS; (e) e (f) GRELL. 
concordância entre as três configurações do modelo global, com diferentes esquemas de convecção e os padrões observados nos experimentos de controle.

Para sintetizar e avaliar objetivamente o desempenho do MCGA-CPTEC em relação as análises T126L28 e T170L42, são apresentados nas Tabelas 3 e 4, o índice de correlação (r) e o erro médio quadrático (EMQ) das auto-interações e interações entre os modos horizontais, para as três categorias de modos verticais e região do composto de ZCAS, conforme

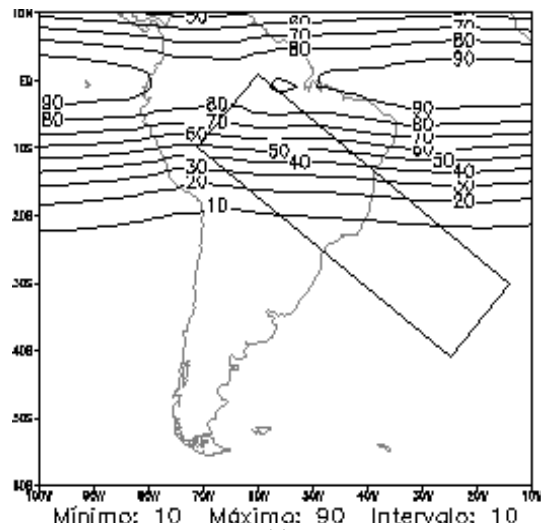

(a)

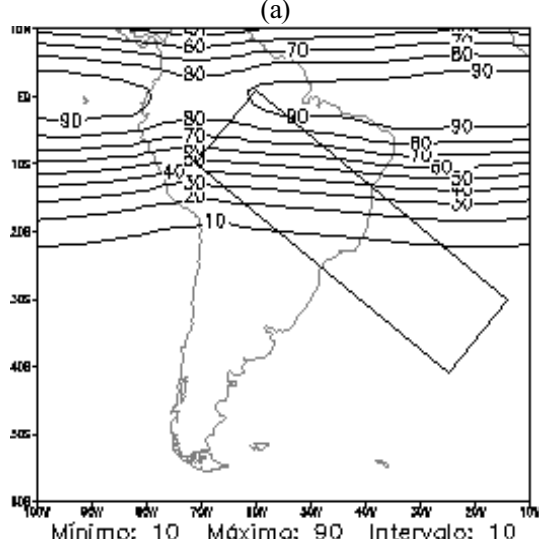

(c)

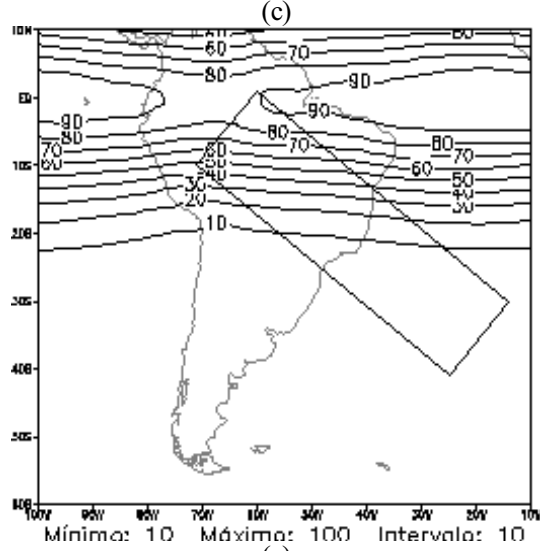

(e) definida na Figura 2. Na primeira coluna das tabelas, a partição de energia total entre os modos horizontais $\mathrm{Ha}$ e $\mathrm{Hb}$ é descrita seguindo a nomenclatura etHaHb. $\mathrm{Ha}$ e $\mathrm{Hb}$ representam os modos horizontais rb (Rossby), kv (Kelvin), mx (Misto), gw (Gravidade Oeste) e ge (Gravidade Leste). No caso das autointerações, $\mathrm{Ha}=\mathrm{Hb}$. As variáveis etnsum e etnres indicam, respectivamente, a soma das auto-interações e das interações entre todos os modos horizontais. Os números escritos na frente de cada variável referem-se aos modos verticais de cada

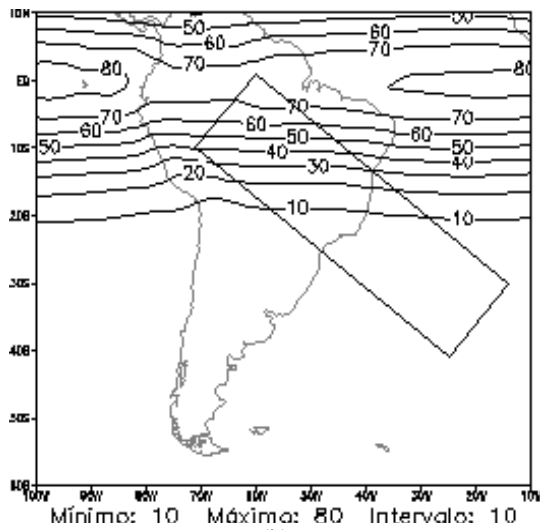

(b)

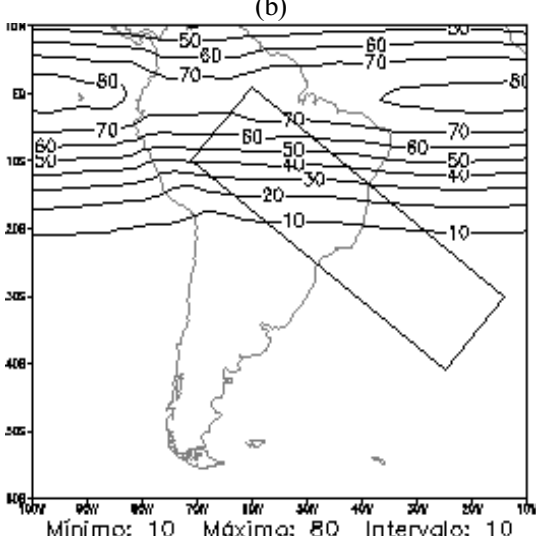

d)

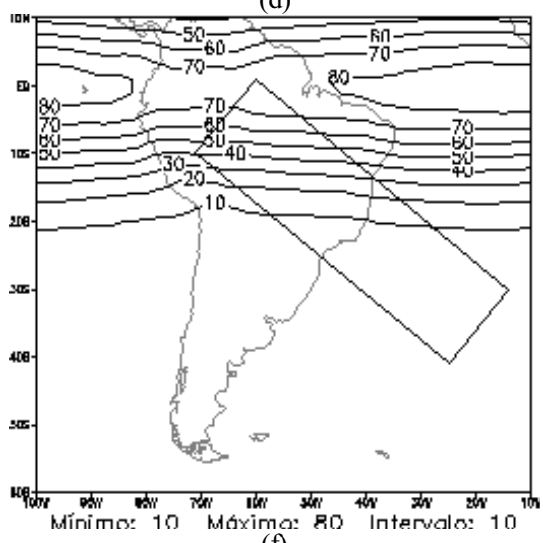

(f)

Figura 10 - Energia Total (\%) do modo horizontal Kelvin para os modos verticais 4 a 7, média das previsões de 24h, MCGA-CPTEC - T126L28 (a esquerda) e T170L42 (a direita): (a) e (b) KUO; (c) e (d) RAS; (e) e (f) GRELL. 


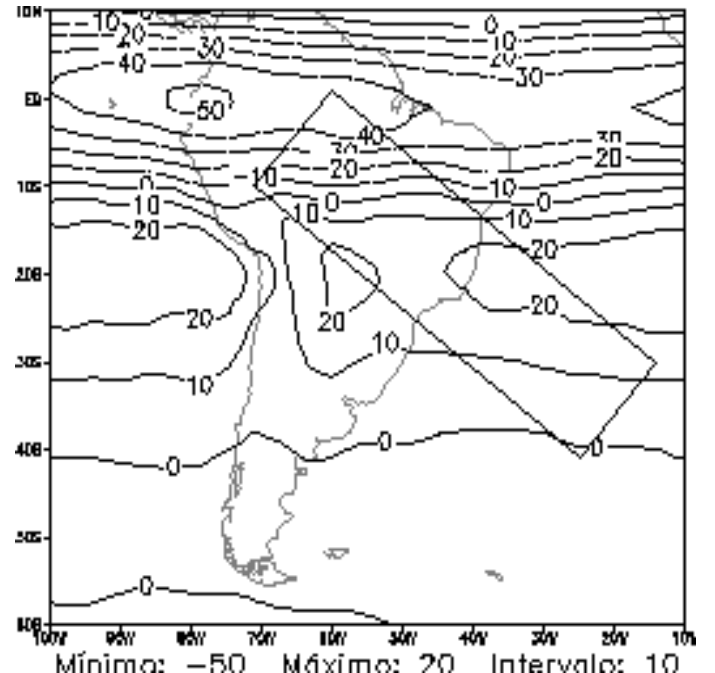

(a)

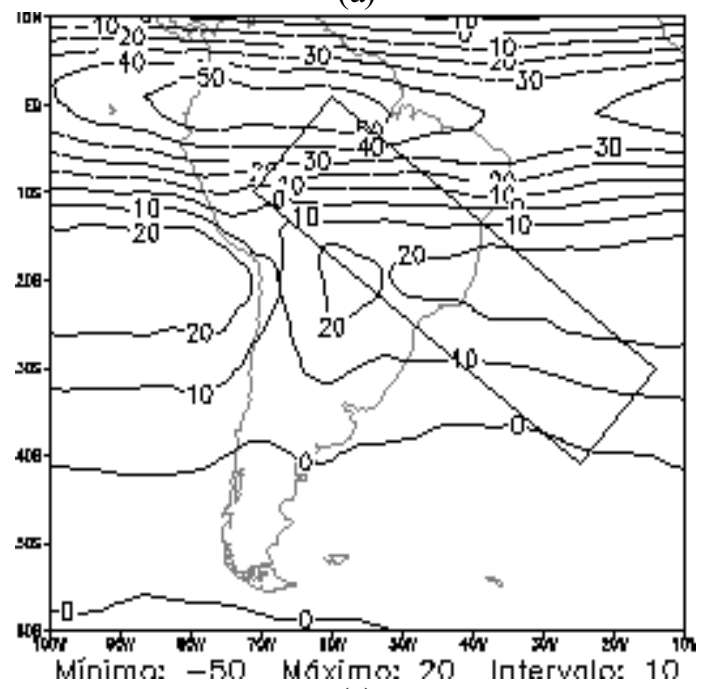

(c)

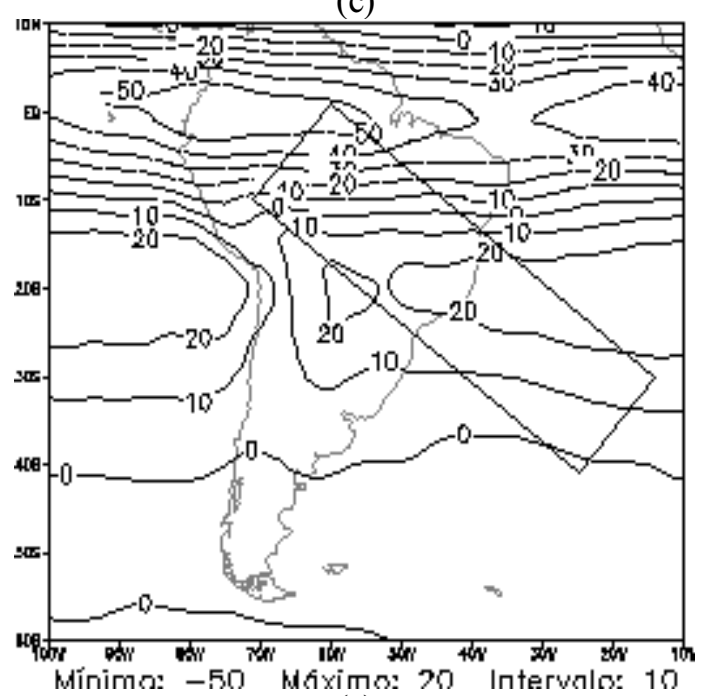

(e)

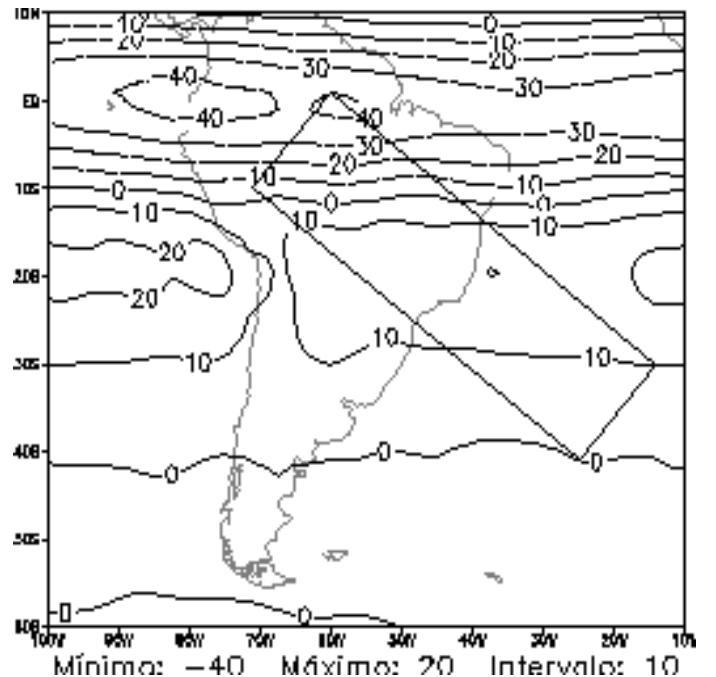

(b)

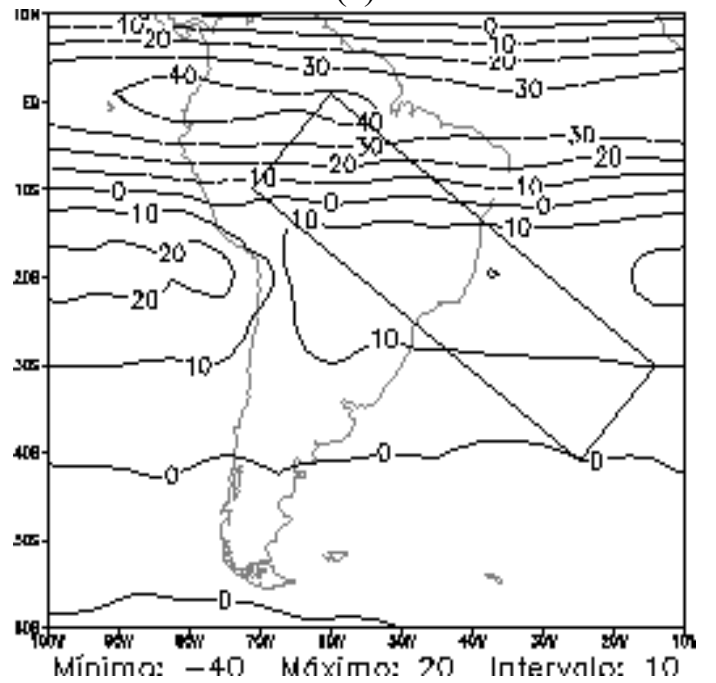

(d)

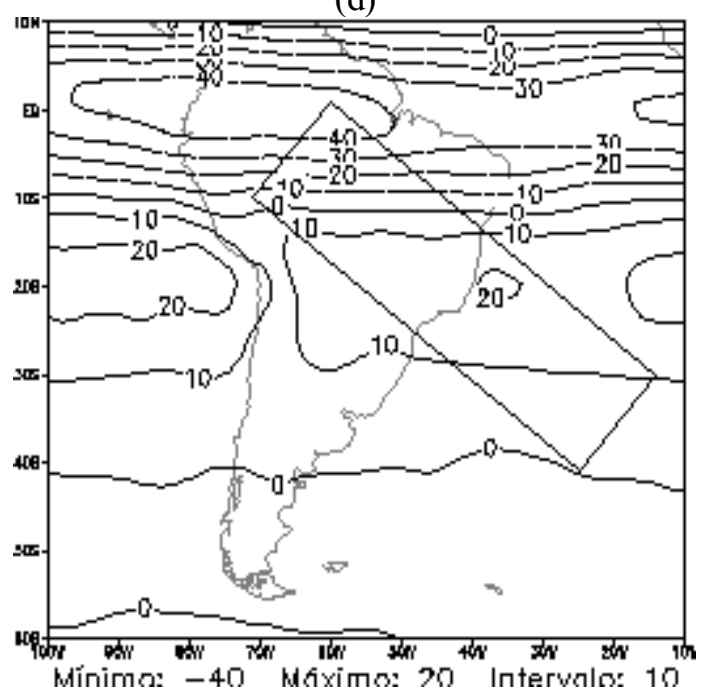

(f)

Figura 11 - Energia Total (\%) da soma de todas as interações - Resíduo, para os modos verticais 4 a 7, média das previsões de 24h, MCGA-CPTEC - T126L28 (a esquerda) e T170L42 (a direita): (a) e (b) KUO; (c) e (d) RAS; (e) e (f) GRELL. 


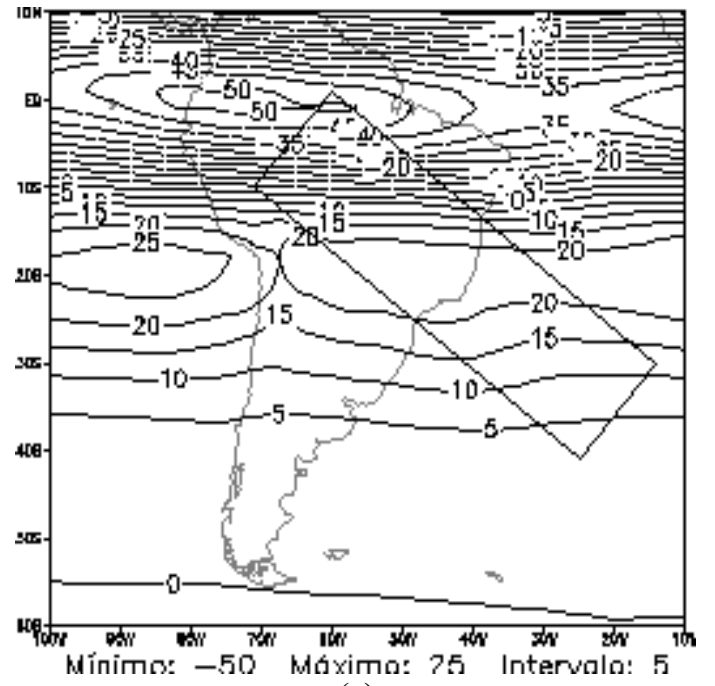

(a)

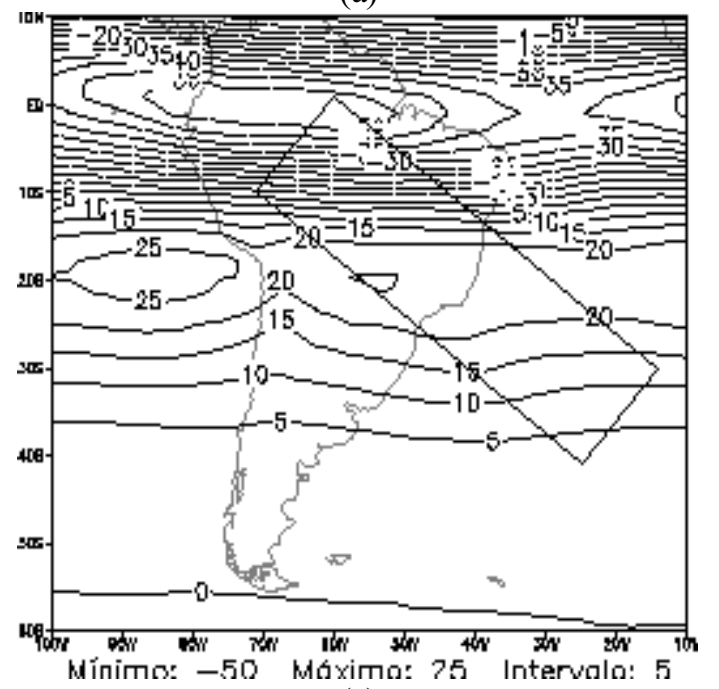

(c)

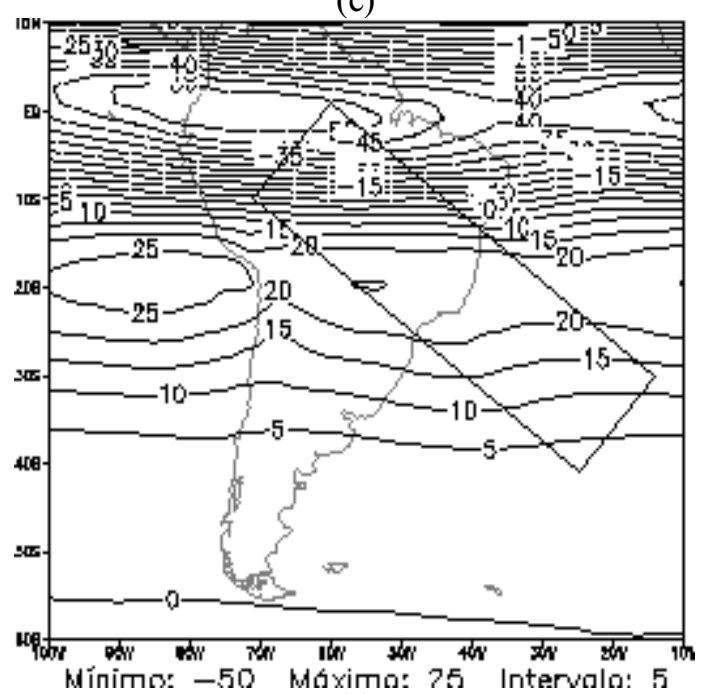

(e)

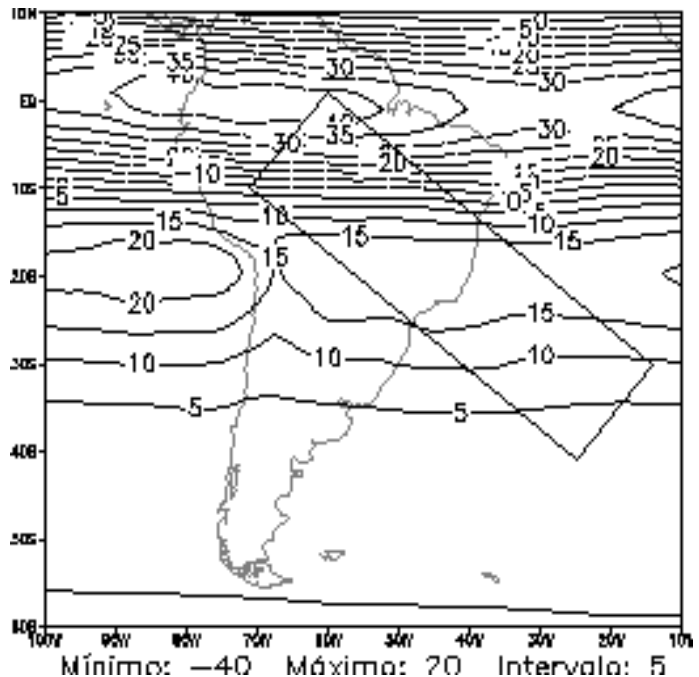

(b)

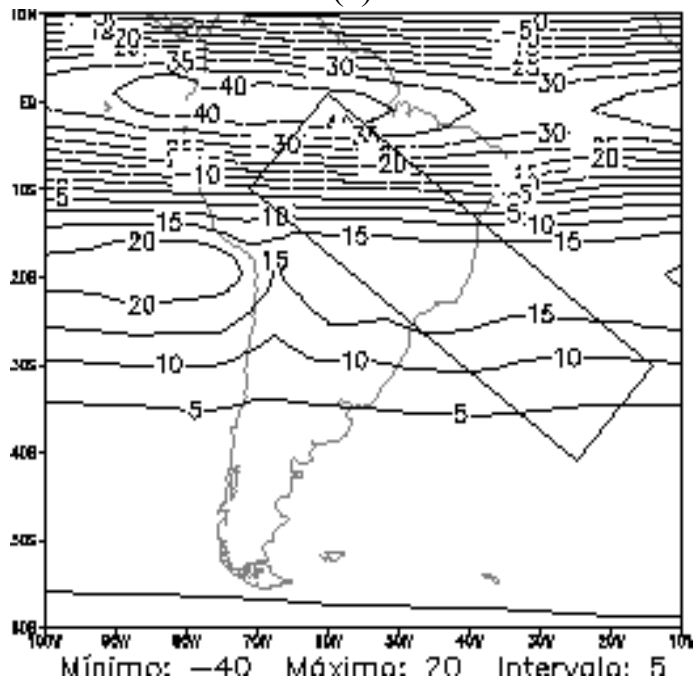

(d)

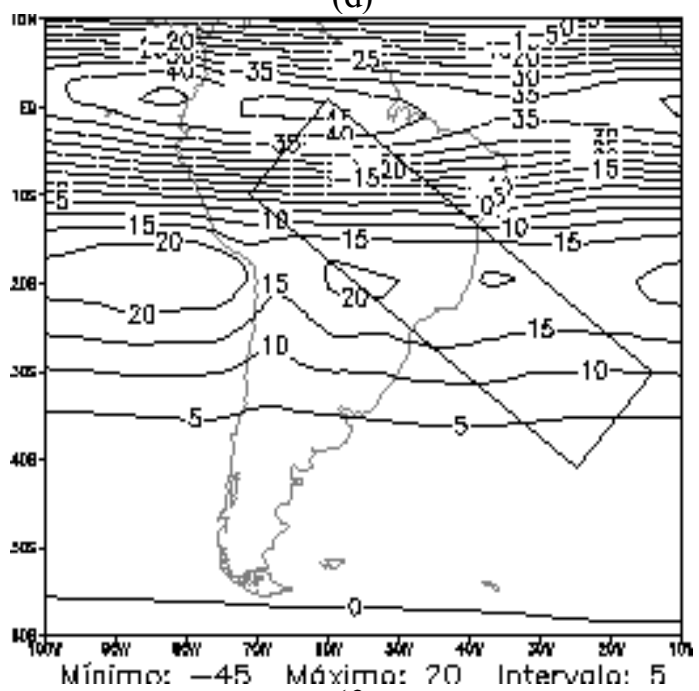

(f)

Figura 12 - Energia Total (\%) das interações entre os modos Rossby e Kelvin, para os modos verticais 4 a 7, média das previsões de 24h, MCGACPTEC - T126L28 (a esquerda) e T170L42 (a direita): (a) e (b) KUO; (c) e (d) RAS; (e) e (f) GRELL. 
categoria. Os valores sublinhados indicam os valores de máxima correlação entre a análise e a previsão; no caso de correlações iguais, considerou-se aquele com menor erro médio quadrático.

Observa-se nas Tabelas 3 e 4, que em geral, os índices de correlação apresentam valores relativamente altos (em torno 0.90) e similares para os três esquemas de convecção profunda, o que confirma o bom desempenho dos esquemas KUO, RAS e GRELL em prever os padrões observados nas Análises T126L28 e T170L42, além da semelhança entre as previsões utilizando estas três parametrizações.
Uma rápida análise dos valores sublinhados indica que o esquema KUO apresenta um desempenho ligeiramente melhor para o modelo T126L28, na região do composto de ZCAS. Resultados similares (não apresentados) também foram obtidos considerando toda a região da América do Sul. No caso do modelo com resolução T170L42, as previsões utilizando o esquema RAS apresentam correlações ligeiramente menores, enquanto o modelo com o esquema GRELL apresenta uma melhor destreza, quando restringimos a área do cálculo dos índices para a região do composto de ZCAS. Um aspecto

Tabela 3 - Índice de Correlação (r) e Erro Médio Quadrático (EMQ) - Energética dos Modos Horizontais - Modelos T126L28 - Região: Composto ZCAS

\begin{tabular}{|c|c|c|c|c|c|c|}
\hline \multirow{2}{*}{$\begin{array}{c}\text { MODO } \\
\text { HORIZONTAL }\end{array}$} & \multicolumn{2}{|c|}{ KUO } & \multicolumn{2}{|c|}{ RAS } & \multicolumn{2}{|c|}{ GRELL } \\
\hline & $\mathbf{r}$ & EMQ $(\%)$ & $\mathbf{r}$ & $\mathrm{EMQ}(\%)$ & $\mathbf{r}$ & $\operatorname{EMQ}(\%)$ \\
\hline etrbrb13 & 0.998 & 0.919 & 0.998 & 0.877 & 0.997 & 0.810 \\
\hline etrbrb 47 & 0.989 & 1.670 & $\overline{0.979}$ & 1.535 & 0.977 & 1.593 \\
\hline etrbrb8 12 & $\overline{0.971}$ & 0.510 & 0.980 & 0.374 & 0.976 & 0.373 \\
\hline etkvkv13 & 0.996 & 0.328 & $\overline{0.973}$ & 0.376 & 0.975 & 0.314 \\
\hline et.kvkv47 & 1.000 & 1.117 & 0.999 & 1.234 & 1.000 & 1.066 \\
\hline etkvkv812 & 0.998 & 0.095 & 0.998 & 0.221 & 1.000 & 0.054 \\
\hline etmumxil3 & 0.915 & 0.014 & 0.908 & 0.011 & $\overline{0.891}$ & 0.012 \\
\hline etmxmx 47 & $\overline{0.954}$ & 0.016 & 0.973 & 0.013 & 0.948 & 0.016 \\
\hline etmxmx 812 & 0.998 & 0.002 & 0.992 & 0.009 & 0.995 & 0.009 \\
\hline etgrom ${ }^{\prime} 13$ & $\overline{0.992}$ & 0.012 & 0.980 & 0.019 & 0.982 & 0.016 \\
\hline etgring 47 & 0.923 & 0.037 & 0.882 & 0.076 & 0.849 & 0.106 \\
\hline etgurgur 812 & $\overline{0.731}$ & 0.022 & 0.739 & 0.035 & 0.773 & 0.036 \\
\hline etgege13 & 0.994 & 0.010 & 0.993 & 0.009 & $\overline{0.990}$ & 0.010 \\
\hline etgege 47 & 0.922 & 0.044 & 0.867 & 0.079 & 0.886 & 0.083 \\
\hline etgege 812 & 0.902 & 0.017 & 0.874 & 0.022 & 0.896 & 0.017 \\
\hline etnsuml3 & 0.997 & 1.123 & 0.998 & 1.108 & 0.997 & 1.009 \\
\hline et.nsum4 7 & 0.998 & 2.436 & 0.998 & 1.456 & 0.998 & 1.387 \\
\hline etnsum812 & 0.936 & 0.535 & 0.939 & 0.536 & 0.956 & 0.398 \\
\hline etnres13 & 0.996 & 0.331 & 0.996 & 0.498 & 0.995 & 0.548 \\
\hline etnres47 & $\overline{0.998}$ & 2.314 & 0.998 & 1.369 & 0.998 & 1.434 \\
\hline etnres812 & 0.982 & 0.193 & 0.979 & 0.223 & 0.979 & 0.231 \\
\hline etrbkv13 & 0.998 & 0.452 & 0.996 & 0.405 & 0.996 & 0.420 \\
\hline etrbkv 47 & 1.000 & 0.991 & 1.000 & 1.448 & 1.000 & 1.468 \\
\hline etrbkv812 & 0.998 & 0.078 & 0.996 & 0.152 & 0.998 & 0.084 \\
\hline etrbomxl 3 & 0.980 & 0.079 & 0.965 & 0.098 & 0.956 & 0.115 \\
\hline etrbomx 47 & $\overline{0.715}$ & 0.238 & 0.860 & 0.227 & 0.828 & 0.206 \\
\hline etrbomx812 & 0.960 & 0.010 & 0.885 & 0.015 & 0.957 & 0.009 \\
\hline etrbgri 13 & 0.998 & 0.206 & 0.997 & 0.249 & 0.997 & 0.226 \\
\hline etrbogr 47 & 0.354 & 1.407 & 0.555 & 1.292 & 0.515 & 1.359 \\
\hline etrbgri 812 & 0.785 & 0.118 & 0.798 & 0.103 & 0.827 & 0.127 \\
\hline etrbgel 3 & 0.999 & 0.142 & 0.999 & 0.114 & 0.998 & 0.112 \\
\hline etrbge 47 & 0.960 & 0.469 & 0.908 & 0.996 & 0.941 & 0.771 \\
\hline etrbge 812 & $\overline{0.801}$ & 0.101 & 0.851 & 0.106 & 0.840 & 0.098 \\
\hline etkvmx13 & 0.965 & 0.093 & 0.976 & 0.088 & 0.973 & 0.093 \\
\hline etkvmx 47 & 0.206 & 0.670 & 0.576 & 0.560 & 0.513 & 0.515 \\
\hline etkvmx 812 & 0.957 & 0.019 & 0.845 & 0.027 & 0.915 & 0.014 \\
\hline et.kvgrw13 & 0.999 & 0.034 & 0.998 & 0.036 & 0.997 & 0.041 \\
\hline etkvgw 47 & $\overline{0.165}$ & 0.543 & 0.347 & 0.288 & 0.144 & 0.407 \\
\hline etkvgु 812 & 0.386 & 0.024 & $\overline{0.691}$ & 0.030 & 0.709 & 0.017 \\
\hline etkvgel 3 & 0.994 & 0.094 & 0.994 & 0.073 & $\overline{0.991}$ & 0.090 \\
\hline et.kvge 47 & 0.711 & 0.943 & $\overline{0.651}$ & 1.196 & 0.716 & 1.363 \\
\hline etkvge 812 & 0.701 & 0.051 & 0.719 & 0.094 & $\overline{0.715}$ & 0.070 \\
\hline etmxgni 13 & 0.950 & 0.007 & 0.954 & 0.009 & 0.948 & 0.010 \\
\hline etmxgrw 47 & 0.733 & 0.015 & 0.595 & 0.017 & 0.494 & 0.024 \\
\hline etmxgrw 812 & $\overline{0.681}$ & 0.005 & 0.818 & 0.005 & 0.690 & 0.007 \\
\hline etmxge 13 & 0.943 & 0.019 & 0.935 & 0.020 & 0.934 & 0.021 \\
\hline etmxge 47 & $\overline{0.875}$ & 0.029 & 0.926 & 0.040 & 0.900 & 0.033 \\
\hline etmxge 812 & 0.655 & 0.009 & 0.316 & 0.011 & 0.282 & 0.011 \\
\hline etgrige13 & 0.989 & 0.022 & 0.977 & 0.027 & 0.977 & 0.025 \\
\hline etgrige 47 & $\overline{0.877}$ & 0.054 & 0.886 & 0.060 & 0.859 & 0.074 \\
\hline etgrifge 812 & 0.880 & 0.012 & 0.817 & 0.015 & 0.838 & 0.014 \\
\hline
\end{tabular}


interessante é que, em ambas as resoluções, o esquema GRELL tende a ser superior para as auto-interações/interações entre modos horizontais na categoria III, ou seja, para os modos internos 8 a 12 e 8 a 14, respectivamente.

\subsubsection{Interação da Energia Total entre os Modos Verticais em Diferentes Níveis de Pressão}

O impacto dos esquemas de convecção profunda na interação de energia entre os modos verticais é discutido a seguir, considerando-se apenas as categorias de modos verticais que apresentaram as maiores porcentagens de energia em cada classe de níveis de pressão. Os resultados para a região do composto de ZCAS são sintetizados nas Tabelas 5 e 6 . $\mathrm{Na}$ primeira coluna das tabelas são descritas a partição de energia total e as interações entre os modos de cada categoria de modos verticais identificadas por MiMf, em que Mi e Mf representam, respectivamente, os modos inicial e final de cada categoria, e para cada classe de pressão x (a - Baixos Níveis, b - Níveis Médios, c- Níveis Altos e d - Estratosfera), seguindo

Tabela 4 - Índice de Correlação e Erro Médio Quadrático (EMQ) - Energética dos Modos Horizontais - Modelos T170L42 - Região: Composto ZCAS

\begin{tabular}{|c|c|c|c|c|c|c|}
\hline \multirow{2}{*}{$\begin{array}{c}\text { MODO } \\
\text { HORIZONTAL }\end{array}$} & \multicolumn{2}{|c|}{ KUO } & \multicolumn{2}{|r|}{ RAS } & \multicolumn{2}{|c|}{ GRELL } \\
\hline & $\mathbf{r}$ & EMQ $(\%)$ & $r$ & EMQ $(\%)$ & $\mathbf{r}$ & EMQ (\%) \\
\hline etrbrb13 & 0.998 & 0.586 & 0.997 & 0.553 & 0.997 & 0.477 \\
\hline etrbrb47 & $\overline{0.991}$ & 1.535 & 0.992 & 1.427 & 0.978 & 1.208 \\
\hline etrbrb814 & 0.991 & 0.684 & 0.993 & 0.609 & 0.995 & 0.391 \\
\hline etkvkv13 & 0.999 & 0.694 & 0.999 & 0.600 & 0.994 & 0.433 \\
\hline etkvkv47 & 1.000 & 1.162 & 1.000 & 1.137 & 1.000 & 0.897 \\
\hline etkvkv814 & 0.999 & 0.151 & 0.999 & 0.146 & 1.000 & 0.068 \\
\hline etmxmx13 & 0.938 & 0.012 & 0.951 & 0.011 & 0.927 & 0.011 \\
\hline $\operatorname{etm} x \min 47$ & 0.938 & 0.013 & 0.940 & 0.014 & 0.942 & 0.013 \\
\hline etmxmx814 & 0.996 & 0.002 & 0.996 & 0.003 & 0.994 & 0.007 \\
\hline etgwgw13 & 0.988 & 0.017 & 0.986 & 0.018 & 0.973 & 0.021 \\
\hline etgwgw 47 & 0.890 & 0.025 & 0.895 & 0.024 & 0.809 & 0.063 \\
\hline etgrowg 814 & 0.672 & 0.018 & 0.640 & 0.019 & 0.764 & 0.028 \\
\hline etgege13 & 0.994 & 0.010 & 0.994 & 0.009 & 0.995 & 0.007 \\
\hline etgege 47 & 0.945 & 0.021 & 0.938 & 0.023 & 0.868 & 0.051 \\
\hline etgege814 & 0.877 & 0.012 & 0.894 & 0.012 & 0.885 & 0.013 \\
\hline etnsum13 & 0.995 & 1.110 & 0.995 & 0.987 & 0.992 & 0.775 \\
\hline etnsum47 & 0.999 & 2.484 & 0.999 & 2.348 & 0.999 & 1.131 \\
\hline etnsum814 & 0.977 & 0.735 & 0.982 & 0.665 & 0.991 & 0.427 \\
\hline etnres13 & 0.998 & 0.40 .5 & 0.998 & 0.383 & 0.999 & 0.509 \\
\hline etnres 47 & 0.998 & 1.948 & 0.998 & 1.869 & 0.999 & 0.953 \\
\hline etnres814 & 0.994 & 0.287 & 0.995 & 0.284 & 0.994 & 0.295 \\
\hline etrbkv13 & 0.998 & 0.652 & 0.999 & 0.598 & 0.998 & 0.481 \\
\hline etrbkv 47 & 1.000 & 0.882 & 1.000 & 0.818 & 0.999 & 1.151 \\
\hline etrbkv814 & 0.999 & 0.127 & 0.999 & 0.136 & 0.999 & 0.135 \\
\hline etrbmx13 & 0.985 & 0.085 & 0.983 & 0.079 & 0.968 & 0.126 \\
\hline etrlom 47 & 0.729 & 0.172 & 0.761 & 0.158 & 0.809 & 0.160 \\
\hline etrbmx814 & 0.896 & 0.022 & 0.899 & 0.021 & 0.937 & 0.015 \\
\hline etrbgw13 & 0.998 & 0.177 & 0.998 & 0.186 & 0.997 & 0.205 \\
\hline etrlogw 47 & 0.303 & 1.159 & 0.342 & 1.112 & 0.554 & 0.997 \\
\hline etrbgrw814 & 0.899 & 0.151 & 0.906 & 0.144 & 0.921 & 0.147 \\
\hline etrbge13 & 0.999 & 0.137 & 0.999 & 0.133 & 0.999 & 0.080 \\
\hline etrbge 47 & 0.966 & 0.286 & 0.961 & 0.324 & 0.925 & 0.557 \\
\hline etrbge8 14 & 0.904 & 0.136 & 0.913 & 0.132 & 0.911 & 0.130 \\
\hline etkvinx13 & 0.980 & 0.127 & 0.980 & 0.123 & 0.982 & 0.136 \\
\hline etkvmx 47 & 0.169 & 0.514 & 0.273 & 0.456 & $\overline{0.442}$ & 0.431 \\
\hline etkvmx814 & 0.977 & 0.050 & 0.975 & 0.041 & 0.968 & 0.031 \\
\hline etkvgow13 & 0.997 & 0.066 & 0.997 & 0.064 & 0.995 & 0.060 \\
\hline etkvgw4 7 & 0.341 & 0.421 & 0.317 & 0.416 & 0.322 & 0.081 \\
\hline etkvgw814 & 0.655 & 0.033 & 0.624 & 0.034 & 0.618 & 0.028 \\
\hline etkvge13 & 0.992 & 0.180 & 0.992 & 0.167 & 0.994 & 0.123 \\
\hline etkvge47 & 0.626 & 0.736 & 0.655 & 0.662 & 0.590 & 0.988 \\
\hline etkvge814 & 0.833 & 0.082 & 0.853 & 0.079 & 0.842 & 0.086 \\
\hline etmxgw13 & 0.954 & 0.007 & 0.9 .53 & 0.007 & 0.958 & 0.010 \\
\hline $\operatorname{etmxgw4} 47$ & 0.635 & 0.012 & 0.669 & 0.011 & 0.528 & 0.016 \\
\hline etmxgw8 14 & 0.811 & 0.004 & 0.825 & 0.004 & 0.756 & 0.005 \\
\hline etmxge13 & 0.947 & 0.017 & $\overline{0.954}$ & 0.016 & 0.948 & 0.017 \\
\hline etmxge47 & 0.864 & 0.022 & 0.901 & 0.019 & 0.909 & 0.023 \\
\hline etmxge814 & 0.550 & 0.007 & 0.603 & 0.007 & 0.520 & 0.009 \\
\hline etgrowge13 & 0.984 & 0.028 & 0.983 & 0.029 & 0.975 & 0.029 \\
\hline etgwge 47 & 0.808 & 0.035 & 0.824 & 0.034 & 0.815 & 0.044 \\
\hline etgwge814 & 0.867 & 0.010 & 0.866 & 0.010 & 0.821 & 0.011 \\
\hline
\end{tabular}


as nomenclaturas etnMiMfx e intMiMfx, respectivamente. As interações entre os modos verticais de duas categorias distintas A e B para cada classe de pressão $x$, são descritas na tabela como intMiAMfAMiBMfBx $\mathrm{O}$ resíduo das interações entre os 12 (14) primeiros modos verticais do modelo com 28 (42) níveis, para cada classe de pressão, é representado por res $0112 \mathrm{x}$ (res0114x).

Analogamente ao observado para a interação de energia entre os modos horizontais, os resultados dos experimentos de modelagem indicam que o modelo apresenta boa concordância em relação aos padrões observados nos experimentos de controle, independente do esquema de convecção profunda utilizado, uma vez que as previsões geradas com cada esquema são muito similares entre si.

Para a resolução T126L28, verifica-se que o modelo com o esquema GRELL apresenta resultados ligeiramente melhores para a porcentagem de energia total, especialmente para a categoria III de modos verticais e para a Estratosfera. Já para a interação entre modos verticais de uma mesma categoria e entre categorias, há um melhor desempenho do

Tabela 5 - Índice de Correlação (r) e Erro Médio Quadrático (EMQ) - Energética dos Modos Verticais - Modelos T126L28 - Região: Composto ZCAS.

\begin{tabular}{|c|c|c|c|c|c|c|}
\hline \multirow{2}{*}{$\begin{array}{l}\text { VARIÁVEIS / } \\
\text { MODOS VERTICAIS }\end{array}$} & \multicolumn{2}{|l|}{ KUO } & \multicolumn{2}{|c|}{ RAS } & \multicolumn{2}{|c|}{ GRELL } \\
\hline & $\mathbf{r}$ & RMS & $\mathbf{r}$ & RMS & $\mathbf{r}$ & RMS \\
\hline Energia Total $(\%)$ & & & & & & \\
\hline etn0103a & 0.997 & 0.079 & 0.997 & 0.075 & 0.996 & 0.072 \\
\hline etn0407a & 0.992 & 0.097 & 0.995 & 0.078 & 0.994 & 0.096 \\
\hline etn0812a & 0.901 & 0.052 & 0.807 & 0.057 & 0.885 & 0.039 \\
\hline etno103b & 0.997 & 0.188 & 0.997 & 0.179 & 0.996 & 0.172 \\
\hline etno $407 b$ & 0.987 & 0.124 & 0.993 & 0.117 & 0.993 & 0.163 \\
\hline etno812b & $0.929 \quad 0.997$ & 0.150 & 0.867 & 0.142 & 0.898 & 0.089 \\
\hline etno103c & 0.995 & 0.054 & 0.998 & 0.054 & 0.996 & 0.051 \\
\hline etno407c & 0.868 & 0.254 & 0.994 & 0.199 & 0.994 & 0.196 \\
\hline etno8120 & 0.997 & 0.106 & 0.721 & 0.125 & 0.850 & 0.096 \\
\hline etno103d & 0.996 & 0.720 & 0.997 & 0.779 & 0.995 & 0.848 \\
\hline etno407d & 0.879 & 0.988 & 0.993 & 0.725 & 0.995 & 0.607 \\
\hline etno812d & & 0.282 & 0.741 & 0.317 & 0.854 & 0.280 \\
\hline Interação-Modos & 0.996 & & & & & \\
\hline int0103a & 0.992 & 0.074 & 0.996 & 0.097 & 0.993 & 0.092 \\
\hline int0407a & 0.938 & 0.276 & 0.994 & 0.260 & 0.995 & 0.404 \\
\hline int0812a & 0.996 & 0.213 & 0.902 & 0.276 & 0.887 & 0.199 \\
\hline into103b & 0.990 & 0.163 & 0.996 & 0.207 & 0.993 & 0.198 \\
\hline int0407b & 0.713 & 0.219 & 0.995 & 0.181 & 0.995 & 0.276 \\
\hline int0812b & 0.997 & 0.170 & 0.648 & 0.202 & 0.794 & 0.132 \\
\hline int0103c & 0.994 & 0.023 & 0.997 & 0.020 & 0.995 & 0.019 \\
\hline int0407c & 0.954 & 0.336 & 0.992 & 0.366 & 0.993 & 0.329 \\
\hline int0812c & 0.996 & 0.108 & 0.953 & 0.109 & 0.949 & 0.070 \\
\hline $103 d$ & 0.994 & 0.259 & 0.997 & 0.322 & 0.993 & 0.308 \\
\hline int0407d & 0.881 & 0.790 & 0.994 & 0.707 & 0.996 & 0.945 \\
\hline into812d & & 0.288 & 0.761 & 0.384 & $\overline{0.799}$ & 0.299 \\
\hline Interação-Classes & 0.979 & & & & & \\
\hline int01030407a & 0.975 & 0.519 & 0.952 & 0.547 & 0.971 & 0.410 \\
\hline $001030812 a$ & 0.978 & 0.413 & 0.969 & 0.294 & 0.965 & 0.267 \\
\hline int04070812a & 0.969 & 0.871 & 0.971 & 1.188 & 0.961 & 0.869 \\
\hline int01030407b & 0.968 & 0.106 & 0.941 & 0.193 & 0.964 & 0.123 \\
\hline int01030812b & $\overline{0.984}$ & 0.524 & 0.956 & 0.441 & 0.950 & 0.404 \\
\hline int04070812b & 0.977 & 0.599 & 0.972 & 0.750 & 0.975 & 0.817 \\
\hline int01030407c & $\overline{0.842}$ & 0.367 & 0.970 & 0.331 & 0.972 & 0.306 \\
\hline int01030812c & $\overline{0.931}$ & 0.153 & 0.696 & 0.233 & 0.725 & 0.240 \\
\hline into4070812c & 0.948 & 0.668 & 0.745 & 1.256 & 0.868 & 1.347 \\
\hline int01030407d & 0.905 & 0.162 & 0.901 & 0.154 & 0.902 & 0.207 \\
\hline int01030812d & 0.983 & 0.024 & 0.737 & 0.056 & 0.659 & 0.046 \\
\hline int04070812d & & 0.106 & 0.985 & 0.300 & 0.974 & 0.137 \\
\hline Residuo & 0.961 & & & & & \\
\hline res0112a & $\overline{0.967}$ & 0.724 & 0.943 & 1.697 & 0.939 & 1.355 \\
\hline resol12b & 0.986 & 0.688 & 0.929 & 0.818 & 0.946 & 1.156 \\
\hline reso112c & $\overline{0.991}$ & 0.851 & 0.955 & 1.587 & 0.981 & 1.548 \\
\hline reso112d & & 0.529 & 0.993 & 0.555 & 0.992 & 1.045 \\
\hline
\end{tabular}


modelo com o esquema RAS nos Baixos e Médios Níveis, enquanto o modelo com o esquema KUO apresenta uma maior concordância em Altos Níveis e na Estratosfera; o resíduo também é melhor previsto utilizando-se o esquema KUO nos níveis troposféricos.

No caso do modelo T170L42, observa-se que os modelos com o esquema RAS e com o esquema GRELL apresentam um melhor desempenho para a previsão das porcentagens de energia total, sendo que este último apresenta resultados ligeiramente melhores para os Altos Níveis e Estratosfera. Para a interação de energia entre os modos verticais e categorias de modos verticais, previsões ligeiramente melhores foram obtidas com o esquema RAS. O modelo com os esquemas KUO e RAS também apresentaram um melhor desempenho para a previsão do resíduo, especialmente nos Baixos e Médios Níveis, em relação ao esquema GRELL.

Em geral, o modelo com resolução T170L42 apresenta índices de correlações maiores, o que sugere que a resolução

Tabela 6 - Índice de Correlação (r) e Erro Médio Quadrático (EMQ) - Energética dos Modos Verticais - Modelos T170L42 - Região: Composto ZCAS.

\begin{tabular}{|c|c|c|c|c|c|c|}
\hline \multirow{2}{*}{$\begin{array}{l}\text { VARIÁVEIS / } \\
\text { MODOS VERTICAIS }\end{array}$} & \multicolumn{2}{|c|}{ KUO } & \multicolumn{2}{|c|}{ RAS } & \multicolumn{2}{|c|}{ GRELL } \\
\hline & $\mathbf{r}$ & EMQ $(\%)$ & $\mathbf{r}$ & EMQ $(\%)$ & $\mathbf{r}$ & EMQ $(\%)$ \\
\hline \multicolumn{7}{|l|}{ Energia Total (\%) } \\
\hline etn0103a & 0.997 & 0.055 & 0.997 & 0.050 & 0.997 & 0.039 \\
\hline$e \operatorname{tn} 0407 a$ & 0.991 & 0.107 & 0.992 & 0.095 & 0.994 & 0.074 \\
\hline $\operatorname{etn} 0814 a$ & 0.896 & 0.055 & 0.914 & 0.050 & 0.953 & 0.030 \\
\hline etno103b & 0.997 & 0.142 & 0.997 & 0.129 & 0.997 & 0.100 \\
\hline etno407b & 0.985 & 0.142 & 0.986 & 0.128 & 0.992 & 0.126 \\
\hline etro814b & 0.894 & 0.166 & 0.911 & 0.151 & 0.964 & 0.071 \\
\hline etnol03e & 0.997 & 0.041 & 0.997 & 0.037 & 0.997 & 0.030 \\
\hline $\operatorname{etn} 0407 \mathrm{c}$ & 0.994 & 0.329 & 0.994 & 0.292 & 0.993 & 0.183 \\
\hline $\operatorname{etn} 0814 \mathrm{c}$ & 0.921 & 0.165 & 0.935 & 0.150 & 0.965 & 0.093 \\
\hline$e \operatorname{tn} 0103 d$ & 0.996 & 0.857 & 0.996 & 0.754 & 0.996 & 0.670 \\
\hline etno407d & 0.994 & 1.211 & 0.995 & 1.066 & 0.997 & 0.458 \\
\hline $\operatorname{etn} 0814 \mathrm{~d}$ & 0.940 & 0.467 & 0.952 & 0.428 & 0.963 & 0.378 \\
\hline \multicolumn{7}{|l|}{ Interação-Modos } \\
\hline int0103a & 0.995 & 0.046 & 0.995 & 0.036 & 0.993 & 0.082 \\
\hline int0407a & 0.993 & 0.332 & 0.994 & 0.278 & 0.996 & 0.278 \\
\hline int0814a & 0.973 & 0.324 & 0.978 & 0.317 & 0.963 & 0.264 \\
\hline int $0103 b$ & 0.995 & 0.110 & 0.995 & 0.086 & 0.993 & 0.191 \\
\hline int0407b & 0.993 & 0.280 & 0.994 & 0.241 & 0.996 & 0.226 \\
\hline into814b & 0.863 & 0.232 & 0.856 & 0.224 & 0.840 & 0.336 \\
\hline int0103c & 0.997 & 0.016 & 0.997 & 0.013 & 0.996 & 0.016 \\
\hline int0407c & 0.995 & 0.566 & 0.995 & 0.484 & 0.995 & 0.368 \\
\hline int $0814 \mathrm{C}$ & 0.761 & 0.172 & 0.762 & 0.159 & $\overline{0.874}$ & 0.114 \\
\hline into103d & 0.995 & 0.171 & 0.996 & 0.135 & 0.993 & 0.288 \\
\hline int0407d & 0.994 & 1.171 & 0.995 & 0.995 & 0.996 & 0.854 \\
\hline into814d & 0.972 & 0.427 & 0.973 & 0.433 & $\overline{0.953}$ & 0.628 \\
\hline \multicolumn{7}{|l|}{ Interação-Classes } \\
\hline int $01030407 a$ & 0.986 & 0.465 & 0.987 & 0.428 & 0.978 & 0.391 \\
\hline int01030814a & 0.982 & 0.703 & 0.985 & 0.664 & 0.979 & 0.392 \\
\hline int04070814a & 0.990 & 0.685 & 0.991 & 0.709 & 0.985 & 0.715 \\
\hline int $01030407 \mathrm{~b}$ & 0.964 & 0.135 & 0.965 & 0.125 & 0.960 & 0.114 \\
\hline int $01030814 \mathrm{~b}$ & 0.975 & 0.908 & 0.979 & 0.867 & 0.966 & 0.634 \\
\hline int $04070814 \mathrm{~b}$ & 0.986 & 0.407 & 0.990 & 0.359 & 0.982 & 0.686 \\
\hline int01030407o & 0.985 & 0.421 & 0.986 & 0.400 & 0.974 & 0.417 \\
\hline int010308140 & 0.877 & 0.256 & 0.877 & 0.255 & 0.725 & 0.335 \\
\hline int040708140 & 0.934 & 0.408 & 0.942 & 0.434 & 0.864 & 0.959 \\
\hline int01030407d & 0.963 & 0.102 & 0.966 & 0.100 & 0.970 & 0.130 \\
\hline int $01030814 \mathrm{~d}$ & 0.884 & 0.039 & 0.866 & 0.042 & $\overline{0.524}$ & 0.054 \\
\hline int $04070814 d$ & $\overline{0.972}$ & 0.166 & 0.974 & 0.149 & 0.934 & 0.318 \\
\hline \multicolumn{7}{|l|}{ Residuo } \\
\hline resol14a & 0.961 & 0.714 & 0.955 & 0.667 & 0.927 & 1.130 \\
\hline reso114b & 0.926 & 0.919 & 0.925 & 0.855 & 0.885 & 1.379 \\
\hline res0114c & 0.985 & 1.110 & 0.988 & 1.039 & 0.987 & 1.124 \\
\hline reso114d & 0.993 & 0.693 & 0.993 & 0.618 & 0.996 & 1.351 \\
\hline
\end{tabular}


vertical das análises e dos modelos tem um maior impacto na partição modal de energia, do que a resolução horizontal e o esquema de convecção utilizado. Além disso, quando o cálculo da destreza do modelo é restrito à região do composto de ZCAS, há uma significativa melhora das previsões de 24 horas utilizando-se o modelo com esquema GRELL e resolução T170L42.

\section{CONCLUSÕES}

O desempenho do modelo de circulação geral (MCGA) do Centro de Previsão de Tempo e Estudos Climáticos (CPTEC) em reproduzir a partição vertical e horizontal de energia, utilizando a técnica de decomposição em modos normais, é avaliado neste estudo para um composto de 7 episódios de Zona de Convergência do Atlântico Sul. Enfatizou-se nesta avaliação o impacto do aumento da resolução do modelo e a utilização de três diferentes esquemas de convecção profunda: KUO, RAS e GRELL.

Os resultados dos experimentos de modelagem com diferentes esquemas de convecção profunda mostraram que as previsões utilizando os esquemas KUO, RAS e GRELL são bastante semelhantes entre si e têm boa concordância com os padrões obtidos nos experimentos de controle dados em Parte I deste artigo (MB08), não apresentando grandes impactos na partição e interação de energia entre os modos verticais e horizontais. Em geral, o modelo com os esquemas KUO e RAS, apresentou as maiores correlações, respectivamente para as resoluções T126L28 e T170L42. O modelo com o esquema GRELL apresentou melhor desempenho nas previsões para os modos verticais 8 a 12 (14, para T170L42), além de apresentar, local ou regionalmente, um melhor detalhamento dos padrões observados.

Desta forma, nota-se que o aumento da resolução vertical do modelo é importante para a representação das características de grande escala dos episódios de ZCAS, independentemente dos esquemas de convecção profunda utilizados neste estudo. Experimentos avaliando o impacto de outros esquemas de convecção profunda mais sofisticados, bem como a influência de outros processos físicos, tais como radiação, convecção rasa, etc. nas trocas horizontais e verticais de energia serão investigados em trabalhos futuros.

\section{AGRADECIMENTOS}

Os autores agradecem ao revisor anônimo da RBMET pelos comentários e sugestões, importantes para o aprimoramento científico deste trabalho. Os autores foram parcialmente financiados pela Coordenação de Aperfeiçoamento de Nível Superior (CAPES). Este trabalho é parte da tese de doutorado do primeiro autor.

\section{REFERÊNCIAS BIBLIOGRÁFICAS}

ANTHES, R.A. A cumulus parameterization scheme utilizing a one-dimensional cloud model. Mon. Wea. Rev., v. 105, n. 3, p. 270-300, 1977.

ARAKAWA, A.; SCHUBERT, W.H. Interaction of a cumulus cloud ensemble with the large-scale envirnment. Part I. J. Atmos. Sci., v. 31, n. 3, p. 674-701, 1974.

BETTS, A.K. A new convective adjustment scheme. Part I: observational and theoretical basis. Quart. J. Roy. Meteor. Soc., v. 112, n. 473, p. 677-692, 1986.

BETTS, A.K.; MILLER, M.J. A new convective adjustment scheme. Part II: single column tests using GATE wave, BOMEX, ATEX and artic air-mass data sets. Quart. J. Roy. Meteor. Soc., v. 112, n. 473, p. 693-709, 1986.

BONATTI, J. P. Modelo de circulação geral do CPTEC. Climanálise Especial 10 anos, v.11, n. especial, out. 1996. Disponível em: http://www.cptec.inpe.br/products/ climanalise/cliesp10a/bonatti.html. Acesso em: $08 \mathrm{fev}$ 2002.

CARVALHO, L.M.V.; JONES C.; LIEBMANN B. The South Atlantic Convergence Zone: intensity, form persistence, and relationships with intraseasonal to interannual activity and extreme rainfall. J. Climate, v. 17, n. 1, p.88-108, 2004.

CHO, H.R.; OGURA, Y. A relationship between the cloud activity and the low-level convergence as observed in ReedRecker's composite easterly waves. J. Atmos. Sci., v. 31, n. 8, p. 2058-2065, 1974.

CLIMANÁLISE - Boletim de Monitoramento e Análise Climática, v.16, n.01, jan, 2001a. Disponível em: http:// www.cptec.inpe.br/products/climanalise/0101/index.html . Acesso em: 17 mar 2003.

CLIMANÁLISE - Boletim de Monitoramento e Análise Climática, v.16, n.11, nov, 2001b. Disponível em: http:// www.cptec.inpe.br/products/climanalise/1101/index.html . Acesso em: 17 mar 2003.

CLIMANÁLISE - Boletim de Monitoramento e Análise Climática, v.16, n.12, dez, 2001c. Disponível em: http:// www.cptec.inpe.br/products/climanalise/1201/index.html . Acesso em: 17 mar 2003.

CLIMANÁLISE - Boletim de Monitoramento e Análise Climática, v.17, n.02, fev, 2002. Disponível em: http://www. cptec.inpe.br/products/climanalise/0202/index.html . Acesso em: 17 mar 2003.

FIGUEROA, S.N. Estudos dos sistemas de circulação de verão sobre a América do Sul e suas simulações com modelos numéricos. 1997. 190f. (INPE-7121-TDI/672). Tese (Doutorado em Meteorologia) - Instituto Nacional de Pesquisas Espaciais, São José dos Campos, 1997.

FRITSCH, J.M.; CHAPPELL, C.F. Numerical prediction of 
convectively driven mesoscale pressure systems. Part I: convective parameterization. J. Atmos. Sci., v. 37, n. 8, p. 1722-1733, 1980.

GANDU, A.W.; SILVA DIAS, P.L. Impact of tropical heat sources on the South American tropospheric upper circulation and subsidence. J. Geophys. Res., v. 103, n. D6, p. 6001-6015, 1998.

GRELL, G.A. Prognostic evaluation of assumptions used by cumulus parameterization. Mon. Wea. Rev., v. 121, n. 3, p. 764-787, 1993.

GRELL, G. A.; DUDHIA J.; STAUFFER D. R. A Description of the Fifth-Generation Penn State/NCAR Mesoscale Model (MM5). Colorado: NCAR, 1994. 117p. Technical Note (NCAR/TN-398 + STR).

GRIMM, A.M.; SILVA DIAS, P.L. Analysis of tropicalextratropical interactions with influence functions of a barotropic model. J.Atmos. Sci., v. 52, n. 20, p. 3538-3555, 1995.

KAIN, J.S.; FRITSCH, J.M. A one-dimensional entraining/ detraining plume model and its application in convective parameterization.J.Atmos. Sci., v.47, n. 23,p. 2784-2802, 1990.

KASAHARA, A.; PURI, K. Spectral representation of threedimensional global data by expansion in normal mode functions. Mon. Wea. Rev., v. 109, n. 1, p. 37-51, 1981.

KINTER III, J. L. et. al. The COLA atmosphere - biosphere general circulation model. Volume 1: Formulation. Calverton, MA: Center for Ocean-Land-Atmosphere Studies (COLA), 1997. Report n. 51. 44p.

KODAMA, Y.M. Large-scale common features of sub-tropical convergence zones (the Baiu Frontal Zone, the SPCZ, and the SACZ) Part I: characteristics of subropical frontal zones. J Meteor. Soc. Japan, v. 70, n. 4, p. 813-836, 1992.

. Large-scale common features of sub-tropical convergence zones (the Baiu Frontal Zone, the SPCZ, and the SACZ) Part II: conditions of the circulations for generating the STCZs. J Meteor. Soc. Japan, v. 71, n. 5, p. 581-610, 1993.

KREITZBERG, C.W.; PERKEY, D.J. Release of potencial instability. Part I: a sequential plume model within a hydrostatic primitive equation model. J. Atmos. Sci., v. 33, n. 3, p. 456-475, 1976.
KUO, H.L. On formation and intensification of tropical cyclones through latente heat release by cumulus convection. J. Atmos. Sci., v.22, n. 1, p. 40-63, 1965.

Further studies of the parameterization of the influence of cumulus convection on large-scale flow. J. Atmos. Sci., v. 31, n. 5, p. 1232-1240, 1974.

KUO, Y.H.; BRESH, J.F.; CHENG, M.-D; KAIN, J.; PARSONS, D.B.; TAO, W.-K.; ZHANG, D.-L. Summary of a mini workshop on cumulus parameterization for mesoescale models. Bull. Amer. Meteor. Soc., v. 78, n. 3, p. 475-491, 1997.

MALKUS, J.S.; WILLIAMS, R.T. On the interaction between severe storms and large cumulus clouds. Meteor. Monog., v. 5, n. 27, p. 59-64, 1963.

MATSUMOTO, S.; NINOMIYA, K.; AKIYAMA, T. Cumulus activities in relation to the meso-scale convergence field. J. Meteor. Soc. Japan., v. 45, n. 4, p. 292-305, 1967.

MENDONÇA, R.W.B. ; BONATTI, J.P. Estudo da energética modal para episódios de ZCAS. Parte I: Análise Observacional. Rev. Brasileira de Meteorologia, 2008. (Aceito para publicação)

MOORTHI, S.; SUAREZ, M.J. Relaxed Arakawa-Schubert-A parameterization of moist convection for general-circulation models. Mon. Wea. Rev., v. 120, n. 6, p. 978-1002, 1992.

PURI, K. The relationship between convective adjustment, Hadley circulation and normal modes of the ANMRC spectral model. Mon. Wea. Rev., v. 111, n. 1, p. 23-33, 1983.

QUADRO, M.F.L. Estudo de episódios de Zona de Convergência do Atlântico Sul (ZCAS) sobre a América do Sul. 1994. 94f. (INPE-6341-TDI/593) Dissertação (Mestrado em Meteorologia) - Instituto Nacional de Pesquisas Espaciais, São José dos Campos, 1994.

STRAUS, D.M.; HUNTLEY, M.A. Interactions between Moist Heating and Dynamics in Atmospheric Predictability. J. Atmos. Sci., v. 51, n. 3, p.447-464, 1994.

WANG, W.; SEAMAN, N.L. A comparison study of convective parameterization schemes in a mesoscale model. Mon. Wea. Rev., v. 125, n. 2, p. 252-278, 1997. 